\title{
Effect of SCN Lesions on Sleep in Squirrel Monkeys: Evidence for Opponent Processes in Sleep-Wake Regulation
}

\author{
Dale M. Edgar, ${ }^{1,2}$ William C. Dement, ${ }^{2}$ and Charles A. Fuller ${ }^{1}$ \\ 1 Department of Animal Physiology and California Primate Research Center, University of California at Davis, Davis, \\ California 95616 and 'Sleep Research Center, Department of Psychiatry and Behavioral Sciences, Stanford University \\ School of Medicine, Stanford, California 94305
}

\begin{abstract}
Sleep and wakefulness are governed by both the suprachiasmatic nuclei of the hypothalamus (SCN), and a sleep homeostatic process; however, the interaction of these control systems is not well understood. From rodent studies it has been assumed that the SCN promote neither wake nor sleep but gate the homeostatic sleep-promoting process. Yet in humans sleep tendency is lowest during the later waking hours of the day, and sleep duration can be predicted because of the precise circadian timing of waking. Thus in primates, the SCN could assure sleep-wake cycle consolidation by actively promoting or facilitating wakefulness. To evaluate this hypothesis, we examined the sleep-wake and sleep-stage patterns of intact and SCN-lesioned (SCNx) squirrel monkeys maintained in constant light. This diurnal primate has consolidated sleep and wake patterns more similar to man than rodents. Sleep-wake, sleep stages, brain temperature, and drinking circadian rhythms were eliminated, and total sleep time was significantly increased $(4.0 \mathrm{hr}$, $\boldsymbol{P}<0.01$ ) in SCNX monkeys. However, total times in deeper stages of non-rapid eye movement (non-REM; e.g., delta sleep) and REM sleep were not significantly affected by SCN lesions. Increased total sleep time was associated with a reduction in subjective day wake consolidation, as evidenced by substantially shorter wake bout lengths in SCNx monkeys (15 $\pm 6 \mathrm{~min}$ ) as compared to intact monkeys (223 $\pm 10 \mathrm{~min} ; P<0.0001$, ANOVA). These findings show that the SCN influence the regulation of daily total wake and sleep times, and implicate an alternative sleep-wake regulatory model in which an SCN-dependent process actively facilitates the initiation and maintenance of wakefulness and opposes homeostatic sleep tendency during the subjective day in diurnal primates.
\end{abstract}

IKey words: Saimiri sciureus, circadian rhythm, suprachiasmatic nucleus, sleep-wakefulness, body temperature, drinking]

\footnotetext{
Received March 24, 1992; revised Sept. 14, 1992; accepted Sept. 17, 1992.

We thank Dr. D. M. Murakami for histological preparations, R. Snyder and R. Wheatland for computer software development, W. Lipman and M. Heinold for assistance with animal handling and polysomnograph evaluations, and Dr. D. Dijk and W. Seidel for constructive advice on the manuscript. Fxperiments were conducted at the University of California at Riverside, and the University of California Primate Research Center at Davis. Research was supported in part by NASA Grant NAG2-562 and NIH Grant MH41477 to C.A.F., National Research Service Award AG05397 to D.M.E., and NIH Grant AG06490 to W.C.D.

Correspondence should be addressed to Dale M. Edgar, Ph.D., Sleep Disorders and Research Center, Department of Psychiatry and Behavioral Sciences, Stanford University School of Medicine, 701 Welch Road \#2226, Palo Alto, CA 94304.

Copyright (C) 1993 Society for Neuroscience $0270-6474 / 93 / 131065-15 \$ 05.00 / 0$
}

The suprachiasmatic nuclei of the hypothalamus (SCN) are essential for the circadian timing of most physiological and behavioral variables in mammals, including sleep-wakefulness. The intrinsic rhythmic properties of the SCN can be observed in the daily behaviors of animals studied in conditions devoid of time cues (Aschoff, 1960; Richter, 1971), or by recording the electrical or metabolic activity of SCN neurons in vivo and in vitro (Schwartz and Gainer, 1977; Green and Gillette, 1982; Groos and Hendricks, 1982; Newman and Hospod, 1986). The unique circadian rhythm-generating properties of donor animals can also be transferred to arrhythmic recipients using SCN transplantation techniques (Ralph et al., 1990), showing that this neural structure is sufficient for the generation of circadian rhythms. Thermal or electrolytic lesions, which ablate the SCN, effectively eliminate circadian rhythms. In rodents, SCN lesions consistently abolish endogenous circadian rhythms in wheelrunning activity, drinking, feeding, pineal $N$-acetyltransferase, plasma corticosterone, and sleep-wakefulness (for reviews, see Rusak and Zucker, 1979; Moore, 1983; Mistlberger and Rusak, 1989). In the rhesus monkey, circadian rhythms in plasma melatonin are lost following bilateral SCN lesions (Reppert et al., 1981), and drinking and feeding circadian rhythms are eliminated in SCN-lesioned ( $\mathrm{SCNx}$ ) squirrel monkeys (Fuller et al., 1981). However, these studies have provided relatively few clues regarding how the SCN control physiological systems in general, or mediate states of arousal in particular.

Studies of sleep in rodents isolated from light-dark cycles or other temporal cues have shown that SCN lesions eliminate the sleep-wake circadian rhythm without appreciably altering daily total sleep time (Mistlberger et al., 1983). In addition, sleep deprivation produces compensatory sleep in SCNx rats (Mistlberger et al., 1983; Tobler et al., 1983), suggesting that a separate homeostatic mechanism controls arousal states independent of circadian control imposed by the SCN. These findings have been crucial to the formulation of contemporary models hypothesizing possible functional interactions between the homeostatic sleep-promoting process and the circadian timing system (Borbely, 1982; Daan et al., 1984).

Although rodents have important utility in understanding sleep-wake regulation, noteworthy differences between rodent and primate sleep-wake patterns could offer important insights into the functional role of the biological clock in arousal state control. For example, the squirrel monkey, a diurnal new-world primate, typically exhibits only a few daytime sleep episodes (e.g., naps) that occupy about $10 \%$ of the subjective day when entrained to an environmental light-dark cycle, if they nap at all (Edgar, 1986). In contrast, the caged laboratory rat exhibits 
numerous sleep-wake transitions and sleeps $30-40 \%$ of the subjective night (active phase) (Borbely and Neuhaus, 1979; Mistlberger et al., 1983). The consolidated nature of sleep-wake circadian rhythms in monkeys (McNew et al., 1972; Wexler and Moore-Ede, 1985) and humans (cf. Dinges, 1989), the apparent lability of the sleeping state relative to waking, and the highly predictable nature of waking onsets relative to the endogenous timing of the human circadian system (Czeisler et al., 1980) suggest that sleep-wake circadian rhythms in diurnal primates could be the product of a circadian pacemaker-controlled process that initiates and maintains wakefulness. We investigated this possibility by comparing the sleep-wake and sleep-stage organization of intact and SCNx squirrel monkeys. We found that total lesions of the SCN not only eliminated circadian rhythms in sleep stages, brain temperature $\left(T_{\mathrm{BR}}\right)$, and drinking behavior, but substantially increased total sleep time through a marked reduction in subjective day wake consolidation relative to intact animals.

\section{Materials and Methods}

\section{Experimental protocol}

Ten adult male squirrel monkeys (Saimiri sciureus) wcighing 1000$1150 \mathrm{gm}$ (approximately 5-9 years of age) were used in this study. Five animals received bilateral radio-frequency lesions focused in the SCN. The five remaining animals served as controls. Both groups were surgically prepared for chronic sleep and $T_{\mathrm{BR}}$ recording. Each animal was housed unrestrained in an individual cage within a separate, temperature-regulated $\left(27 \pm 0.3^{\circ} \mathrm{C}\right)$, sound-attenuated environmental chamber. These chambers were located in a room free of extraneous sound. Constant light (LL) was maintained throughout this study using broadspectrum fluorescent lamps. Light intensity averaged 500 lux when measured at the level of the animal's head. Food and water were provided ad libitum.

Prior to surgical intervention, the free-running drinking behavior of each animal was monitored for $60 \mathrm{~d}$ while housed in a chronic isolation chamber and maintained in LL. Drinking patterns were also recorded following SCN lesion surgery as a preliminary bioassay of lesion efficacy. Throughout the study, routine cage maintenance (e.g., cleaning, feeding, etc.) was conducted using an alternating 3 and $4 \mathrm{~d}$ schedule.

SCN-lesioned and control animals were permitted $60 \mathrm{~d}$ for postoperative adaptation to $\mathrm{LL}$ in chronic isolation chambers. They were then transferred to the sleep recording chambers where they were permitted an additional $20 \mathrm{~d}$ (in LL) for adaptation to the recording environment prior to polygraph recording of sleep patterns. Brain temperature and drinking frequency were monitored in parallel with polygraph recordings of sleep-wakefulness for a minimum of $5 \mathrm{~d}$. One day before and during all sleep recordings, the animals were completely undisturbed.

\section{Surgical procedures}

$S C N$ lesions. All surgical procedures were conducted under sterile conditions in completely anesthetized animals. Anesthesia was induced with a combination of diazepam (Valium, $0.7 \mathrm{mg} / \mathrm{kg}, \mathrm{i} . \mathrm{m}$.) and sodium pentobarbital (Nembutal, $17 \mathrm{mg} / \mathrm{kg}$, i.p.). After a stable plane of deep anesthesia was established, the animal's head was positioned in a stereotaxic framc (David Kopf Instruments). SCN lesions were performed using a procedure similar to that previously described (Fuller et al., 1981).

Two methods were employed to facilitate accurate lesion electrode placement. First, radiopaque dye (meglumine iothalamate, $10 \mu \mathrm{l}$; Mallinckrodt, Conray) was injected into the lateral ventricle to permit $\mathrm{x}$-ray visualization of the third ventricle. In two animals, visual-evoked potentials were also recorded from the dorsal margin of the optic chiasm in order to locate the ventralmost margin of the SCN. X-ray plates, showing the recording electrode position, and ventriculography plates were used to establish the optimum coordinates for placement of the lesion electrode (Radionics RFG 4A, TC series probe). Initial stereotaxic coordinates of the SCN (A 11.5, L $\pm 0.8, V 0.5 \mathrm{~mm}$ ) were based on those of Emmers and Akert (1963). Additional x-rays were used to verify appropriate final electrode placement prior to lesioning. Two radiofrequency, thermal lesions $\left(68-74^{\circ} \mathrm{C}\right.$ for $2 \mathrm{~min}$ ) were made $1 \mathrm{~mm}$ apart along the rostral-caudal axis of each nucleus. In addition, one of the five control animals served as a surgical sham, in which the electrode was positioned but the SCN were not lesioned. The extent of the craniotomy was then filled with Gelfoam and sealed with bone wax. Immediately following the lesion procedure, and as part of the same surgery, the SCN-lesioned animals were surgically prepared with a chronic sleep recording implant.

Slecp recording implant. The five monkeys that received SCN lesions and the five control animals were surgically prepared with a small cranial implant that permitted electrophysiological correlates of sleep [the electroencephalogram (EEG), electrooculogram (EOG), and electromyogram (EMG)] and $T_{\mathrm{BR}}$ to be monitored continuously (Edgar, 1986). As in the SCN lesion procedure, the sleep implant procedure was performed in completely anesthetized animals positioned in a stereotaxic frame. In brief, a $0.2-\mathrm{mm}$-diameter glass thermistor (Thermometrics, Edison, NJ) mounted to the tip of a 23 gauge stainless steel tube was stereotaxically placed through a $2-\mathrm{mm}$-diameter craniotomy (stereotaxic coordinates: A $6.5, \mathrm{~L} 5, \mathrm{~V} 11-13 \mathrm{~mm}$ ) and positioned between the internal capsule and thalamus. X-rays were used to confirm proper positioning. Inverted stainless steel screws (0-80, Small Parts, Inc.) served as epidural EEG recording electrodes (Sheatz, 1961) and were attached by gold wire leads to a miniature connector (Microtech, Boothwynn, PA). Anterior EEG electrodes were positioned $10 \mathrm{~mm}$ apart bilaterally over the frontal cortex (coordinates A $+15.0 \mathrm{~mm}, \mathrm{~L} \pm 5.0 \mathrm{~mm}$ ). A ground electrode was placed posterior to the connector, left of the sagittal crest (coordinates A $0.0 \mathrm{~mm}, \mathrm{~L} 4.0 \mathrm{~mm}$ ). Dental acrylic was applied to these screws and the connector secured the assembly to the skull. Two posterior EEG electrodes were positioned bilaterally over the parieto-occipital cortices (coordinates $\mathrm{A}-20.0 \mathrm{~mm}, \mathrm{~L} \pm 5.0 \mathrm{~mm}$ ) using small stainless steel screws $(0-80 \times 1 / 8$ inch $)$ and flexible, insulated stainless steel wire. EOG leads were positioned lateral to the orbital canthi and secured in bone with miniature stainless steel screws $(00-90 \times 1 / 8$ inch, Small Parts). EMG leads were constructed of Teflon-insulated multistranded stainless steel wire (Bergen Co., Lodi, NJ) routed subcutaneously to the neck musculature and secured with silk suture. Antibiotics (Flocillin, 40,000 IU/ $\mathrm{kg}, \mathrm{i} . \mathrm{m}$.) were administered to each animal. $\Lambda$ minimum of 1 month for recovery was then permitted prior to the initiation of any experimental protocols.

\section{Data acquisition}

Brain temperature and polygraphic sleep parameters were obtained from animals via a flexible cable and commutator assembly that permitted complete freedom of movement within the cage. The monkeys were conditioned to accept the cable during $L L$ adaptation prior to sleep and temperature recording. A video camera was used to monitor the animals condition within the sleep recording chambers. The cable did not appear to aggravate the animal or cause other behavioral disturbances. EEG, EOG, and EMG activities were amplified and recorded using a Grass polygraph (model 78D) and P5 11 amplifiers. EEG and EOG activity were bandpass filtered $(0.3-30 \mathrm{~Hz})$. EMG was bandpass filtered at 10 $90 \mathrm{~Hz}$. EEG activity was recorded using three bipolar electrode configurations: bilaterally across the parieto-occipital cortices, across the frontal cortices, and along the rostral-caudal axis of the frontal and parietooccipital cortices. These data, as well as EOG and EMG activities, were recorded continuously for $120 \mathrm{hr}$ on paper at a chart speed of $5 \mathrm{~mm} /$ sec. Brain temperature was simultaneously recorded at 2 min intervals using an automated data collection system. Data were ultimately transferred to laboratory computers for storage, analyses, and plotting.

\section{Sleep state determinations}

The polygraph records were divided into $30 \mathrm{sec}$ epochs that were scored based on the predominant state. Arousal states were categorized as awake, light slow wave sleep (SWS1), deep slow wave sleep (SWS2), and rapid eye movement sleep (REM). Arousal state determinations were based on standardized criteria described in detail elsewhere (Edgar, 1986) and are similar to that described in other squirrel monkey sleep studies (Adams and Barratt, 1974; Wexler and Moore-Ede, 1985). Awake was characterized by elevated EMG, rapid eye movements, and lowamplitude EEG $(<50 \mu \mathrm{V})$. SWS1 was identified by slow rolling eye movements, low EMG level (compared to awake), and EEG patterns dominated by 4-8 $\mathrm{Hz}$ mixed-amplitude $(75-200 \mu \mathrm{V})$ activity. SWS2 was scored when the EEG activity exhibited high-amplitude $(>75 \mu \mathrm{V})$, low-frequency $(<3 \mathrm{~Hz}$ ) activity during $25 \%$ or more of the epoch. Some slow rolling eye movements were usually observed during SWS2, and EMG was depressed similar to that observed in SWS1. REM sleep was polygraphically characterized by a complete loss of muscle tone with 
intermittent phasic twitches and stereotypic rapid eye movements. EEG activity was dominated by low-amplitude $(50-75 \mu \mathrm{V})$, mixed beta frequency $(>13 \mathrm{~Hz})$ during REM.

Each polygraph record was independently scored by two trained, primate-sleep evaluators. Any record in which the scores of the evaluators showed less than $90 \%$ agreement for any of the four arousal states was rescored jointly by the evaluators.

\section{Histology}

Subsequent to study, the SCN-lesioned squirrel monkeys were given a lethal dose of sodium pentobarbital. The brain was then perfused with normal saline followed by a solution of $2 \%$ paraformaldehyde and $2.5 \%$ glutaraldehyde in $0.1 \mathrm{M}$ phosphate buffer $(\mathrm{pH} 7.6)$. The brain was removed and placed in a solution of $30 \%$ sucrose in $0.1 \mathrm{M}$ phosphate buffer $(\mathrm{pH} \mathrm{7.6)}$ until the brain sunk. The brains were then frozen and cut into $40 \mu \mathrm{m}$ coronal sections, and alternate sections were stained for Nissl substance with thionin, or stained for cytochrome oxidase (Cytox; Wong-Riley, 1979).

Because lesioned areas of the brain have lower metabolic activities than the surrounding neurons, Cytox staining proved a useful tool for estimating the extent of the SCN lesions. Cytox staining was performed by placing alternate brain sections in $0.1 \mathrm{M}$ phosphate buffer $(\mathrm{pH} \mathrm{7.6)}$ and then transferring them to glass vials containing $25 \mathrm{mg}$ of cytochrome C, $50 \mathrm{mg}$ of diaminobenzidine, and $4 \mathrm{mg}$ of sucrose in $100 \mathrm{ml}$ of 0.1 M phosphate buffer adjusted to $\mathrm{pH} 7.6$. The vials were incubated in a temperature-controlled shaker bath $\left(35-37^{\circ} \mathrm{C}\right)$ for approximately $2 \mathrm{hr}$. The vials were then decanted, and the sections rinsed in phosphate buffer before mounting on glass slides.

\section{Data analysis}

Periodicity analysis. Linear-nonlinear least-squares multiple periodic regression analysis (Rummel et al., 1974) was used to detect and quantify periodicity in the data. Periods were assayed from 2 to $38 \mathrm{hr}$ in 0.20 $\mathrm{hr}$ increments, and the criterion for statistical significance of spectral components was set at the 0.05 probability level. This technique has previously been employed to detect periodicity in body temperature and drinking data obtained from both SCN-lesioned and intact squirrel monkeys (Fuller et al., 1981; Gander and Moore-Ede, 1983). Chi-square periodicity analysis (Sokolove and Bushell, 1978) was also applied to the data to validate the loss of circadian rhythms following SCN lesions. For this analysis, periods were assayed from 16 to $32 \mathrm{hr}$ in $0.1 \mathrm{hr}$ increments.

Waveform analysis. Mean circadian waveforms of sleep-wakefulness were computed using a modification of the phase-specific probability analysis technique of Richardson et al. (1985). In brief, the probability of sleep was estimated by dividing the number of occurrences of sleep (SWS1, SWS2, and/or REM) by the total number of scored epochs in each of 48 successive and discrete time bins across each circadian day. Circadian period $(\tau)$ estimates for individual animals were used to define circadian day length in real time. Corresponding probability values were averaged across multiple circadian days, and then across animals, yielding a plot of the population mean phase-specific probability for total sleep. Probabilities were plotted as percentage of time awake per halfhour bin in circadian time (CT) and plotted as a function of circadian time of day. By convention, $\tau$ is divided into 24.0 circadian hours, permitting legitimate waveform averaging across animals with differing circadian period lengths when measured in real time.

Bout length analysis. The longest duration of discrete bouts of wakefulness and of sleep (SWS1 + SWS2 + REM) across the circadian day was computed. A bout was defined as three or more consecutive epochs of a polygraphically defined state. Wake bout lengths were measured within a $4 \mathrm{hr}$ window moved stepwise in $2 \mathrm{~min}$ increments through the sleep-wake time series of each animal. Sleep bout lengths were measured in a $90 \mathrm{~min}$ window moved stepwise in $2 \mathrm{~min}$ intervals. Window size was defined empirically based on the longest wake and sleep bout observed in the population. In each step, the longest sleep bout and longest wake bout within the window were identified and recorded. Bout lengths at corresponding circadian times were averaged across multiple cycles of data for individual animals. Bout lengths were then averaged from animals in corresponding experimental groups and plotted as a function of circadian time (mean \pm SEM).

Phase-specific bout count analysis. Sleep-wake fragmentation was assessed as a function of time of day by counting the number of wake and sleep bouts that occurred within a window moved stepwise through the time series. Window definitions for sleep and wake, and moving incre- ments were identical to that described in bout length analysis (above). Bout counts at corresponding circadian times were averaged for individual animals, and then averaged across corresponding groups to gencrate grand mean phase-specific bout count waveforms. A sleep bout was defined as a continuous series of sleep stages (SWS1, SWS2, or REM) interrupted by not more than one consecutive epoch of wakefulness within a 90 min window. A wake bout was defined in a similar manner with not more than one consecutive epoch of intervening sleep in a 240 min window.

\section{General statistics}

Population statistics were applied to the data $(N=5$ intact monkeys and $N=5$ SCNx monkeys for every statistical application) using Student's $t$ test or analysis of variance (ANOVA). A two-tailed $t$ test was applied to the data and included tests for equal variance, unless specified otherwise. All statistical computations were performed using SAS V6.04 (SAS Institute, Cary, NC). Repeated-measures ANOVA was applied to eight equally spaced time bins per circadian cycle to determine significant differences in the waveforms of intact and SCN-lesioned monkeys. In the presence of a significant main effect the Ryan-Einot-GabrielWelsch (REGW) multiple $F$ test was applied to specific time bins. Significant main effects were further validated by treating each of the eight time bins as dependent variables using multivariate ANOVA. All reported significant main effects showed corresponding significant $(P<$ $0.05)$ Hotelling-Lawley Trace statistics. Averages reported are mean \pm standard error of the mean (SEM).

\section{Results}

\section{Circadian rhythms in intact monkeys}

In LL, intact monkeys exhibited robust free-running circadian rhythms of sleep-wakefulness, $T_{\mathrm{BR}}$, and drinking with periods significantly greater than $24.0 \mathrm{hr}(P<0.01$, one-tailed $t$ test $)$. Periodicity analysis revealed mean periods for intact monkeys as follows: sleep-wake, $25.0 \pm 0.2 \mathrm{hr} ; T_{\mathrm{BR}}, 25.0 \pm 0.2 \mathrm{hr}$; drinking, $25.0 \pm 0.3 \mathrm{hr}$. At no time did the circadian rhythm period of any two variables differ significantly $(P>0.75$, twotailed $t$ test) within a given intact monkey.

A plot of six circadian days of sleep-wake and $T_{\mathrm{BR}}$ data recorded from a representative intact monkey free-running in LL is shown in Figure 1. Consolidated intervals of wakefulness and sleep were observed during the subjective day and night, ${ }^{1}$ respectively, in each monkey. However, rapid transitions between arousal states were common, particularly between wakefulness (WAKE) and SWS1 during the early and late portions of the subjective night. $T_{\mathrm{BR}}$ cycled with an amplitude of approximately $2^{\circ} \mathrm{C}$. The peak of the temperature circadian rhythm corresponded closely with wakefulness and activity behaviors (e.g., drinking).

The REM and SWS2 sleep stages of intact monkeys were more frequently observed when body temperature was below the circadian mean $\left(38.3 \pm 0.3^{\circ} \mathrm{C}\right)$. REM sleep episodes occurred with a period of approximately $58 \mathrm{~min}$, with episodes lasting up to $14 \mathrm{~min}$. During subjective day napping, REM sleep and sleep onset REM periods (i.e., following arousals of $2-3 \mathrm{~min}$ in duration) were occasionally observed in two monkeys but only when $T_{\mathrm{BR}}$ was above the circadian temperature mean. The remaining animals did not show REM during the subjective day. Sleep onset REM was never observed when $T_{\mathrm{BR}}$ was beluw the circadian mean.

The distribution of arousal states for each intact monkey is shown in Table 1. Sleep stage percentages, calculated as a fraction of the circadian total sleep time [CTST; e.g., total sleep in

\footnotetext{
'Subjective day and night are defined here as the predominant waking and rest portions of the circadian day, respectively. More conservatively, the subjective day is defined by the largest consecutive interval in time during the circadian day when body temperature exceeds the rhythm mean.
} 


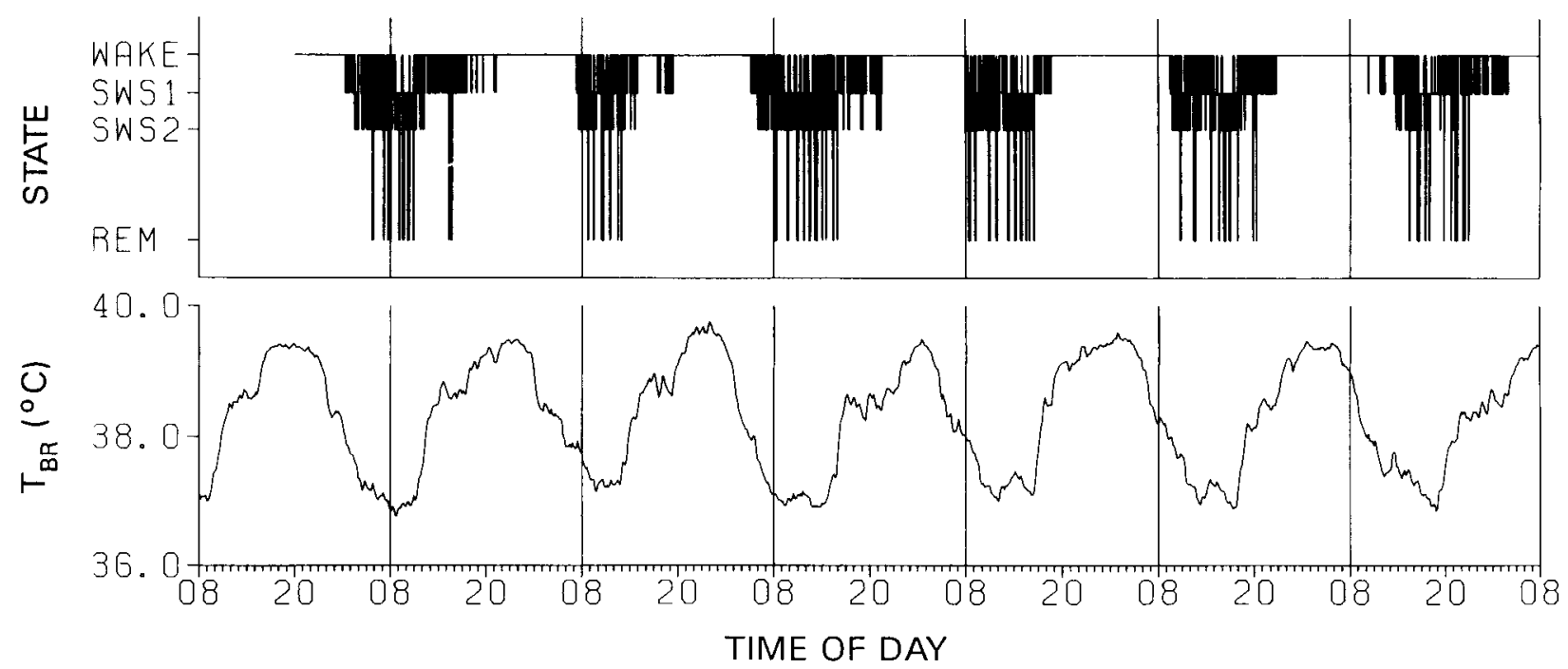

Figure 1. Arousal state and $T_{\mathrm{BR}}$ rhythms monitored simultaneously from a sham-treated squirrel monkey for approximately 6 circadian days $(\tau$ $=25.5)$ in LL ( $500 \mathrm{lux}$ ). Intact and sham-lesioned monkeys showed robust sleep-wake rhythms characterized by highly consolidated bouts of wakefulness and $2^{\circ} \mathrm{C}$ oscillations in $T_{\mathrm{BR}}$. Data shown were obtained from monkey 2 , listed in Table 1 .

hours (real time) per $\tau$ ] were as follows: SWS1, 80.7; SWS2, 10.9; REM, 8.4. On average, $1.7 \mathrm{hr}$ of sleep ( $19 \%$ of CTST) was observed during the subjective day, and consisted primarily of SWS1.

\section{Effect of SCN lesions on rhythmicity}

Circadian rhythms in sleep-wakefulness, $T_{\mathrm{BR}}$, and drinking behavior were absent in all of the SCNx monkeys throughout the study, and remained absent in animals studied up to a year later. Figure 2 shows double raster plots of drinking patterns in an intact and SCNx monkey. The effect of SCN lesions on sleepwake timing and $T_{\mathrm{BR}}$ is shown in Figure 3 . The sleep-wake patterns of SCNx monkeys exhibited frequent transitions between states and numerous arousals similar to the patterns of sleep of intact monkeys during the subjective night (see Fig. 1). Bouts of sleep and wakefulness were generally aggregated in clusters that corresponded with ultradian variations in $T_{\mathrm{BR}}$ and drinking behavior. The periods of these ultradian components averaged $3.8 \mathrm{hr}$ (range, 3.2-4.4 hr). The amplitude of the ultradian variations in $T_{\mathrm{BR}}$ varied between animals. The monkey shown in Figure 3 revealed $T_{\mathrm{BR}}$ variations (peak to peak amplitude of raw data) of $0.75-1.5^{\circ} \mathrm{C}$. In other monkeys, the range of $T_{\mathrm{BR}}$ variations was smaller $\left(0.3-0.7^{\circ} \mathrm{C}\right.$; Fig. 4$)$. There was no obvious correlation between the amplitude of ultradian temperature variations and lesion size or placement (also see His- tological verification, below). The mean, regulated level of $T_{\mathrm{BR}}$ averaged from the five $\mathrm{SCNx}$ monkeys was $38.0 \pm 0.3^{\circ} \mathrm{C}$, which was only $0.3^{\circ} \mathrm{C}$ lower than that observed in the intact monkeys (NS, $P>0.5, t$ test).

Both period analysis procedures (see Materials and Methods) confirmed the loss of circadian rhythms. Figure 5 shows spectral plots for each variable from the sham control and an SCNx monkeys (same animals as in Figs. 1, 3). Each arousal state (awake, SWS1, SWS2, and REM), $T_{\mathrm{BR}}$, and drinking showed a significant $(P<0.05)$ circadian spectral component in every intact monkey. The free-running circadian period for the sham surgery monkey (Fig. 5, left) was 25.5 hr. In contrast, SCNx animals showed no significant spectral component for periods ranging from 18 to $38 \mathrm{hr}$. However, three of five SCNx animals showed significant pcriodicity at around $4 \mathrm{hr}$, particularly for drinking and $T_{\mathrm{BR}}$.

Diminution of the sleep-wake circadian rhythm is further illustrated in the mean waveforms of wakefulness shown for intact and SCNx monkeys in Figure 6. The wakefulness waveform of intact monkeys exhibited an asymmetric sinusoidal waveform, related to the ratio of wakefulness to sleep $(\alpha: \rho)$. For the purposes of describing this ratio, subjective day $(\alpha)$ was defined as the interval over the circadian day [measured in circadian time: 24 circadian hours $\left(\mathrm{hr}_{\mathrm{cl}}\right)$ per $\tau$ ] in which wake occurred more than $50 \%$ time, as assessed in consecutive 0.5

Table 1. Arousal state distribution in intact monkeys

\begin{tabular}{llllllr} 
& \multicolumn{2}{l}{ Mean \% state \pm SD/circadian day } & \multicolumn{2}{c}{$\begin{array}{c}\text { CTST }^{a} \\
\text { Monkey }\end{array}$} & \multicolumn{1}{c}{ (hr) } \\
\cline { 2 - 6 } 1 & 24.5 & $64.8 \pm 3.9$ & $28.6 \pm 3.8$ & $3.7 \pm 0.3$ & $2.9 \pm 0.3$ & 8.6 \\
2 & 25.5 & $67.8 \pm 3.2$ & $24.2 \pm 3.0$ & $4.9 \pm 0.7$ & $3.1 \pm 0.3$ & 8.2 \\
3 & 25.2 & $60.1 \pm 4.4$ & $34.3 \pm 4.3$ & $2.5 \pm 0.6$ & $3.1 \pm 0.2$ & 10.0 \\
4 & 24.8 & $64.4 \pm 3.3$ & $28.0 \pm 3.2$ & $4.8 \pm 0.4$ & $2.8 \pm 0.3$ & 8.8 \\
5 & 25.1 & $64.2 \pm 4.1$ & $29.1 \pm 3.9$ & $3.4 \pm 0.6$ & $3.3 \pm 0.4$ & 8.9 \\
Group \pm SEM & $25.0 \pm 0.2$ & $64.3 \pm 1.2$ & $28.8 \pm 1.6$ & $3.9 \pm 0.4$ & $3.0 \pm 0.1$ & $8.9 \pm 0.3$ \\
\hline
\end{tabular}

${ }^{a}$ CTST, total sleep time (hours in real time) per circadian day length $(\tau)$. 
$\mathrm{hr}_{\mathrm{ct}}$ bins (see mean waveform analysis in Materials and Methods). Intact monkeys demonstrated a mean $\alpha: \rho$ ratio of 14.5 : 9.5 (based on $\mathrm{hr}_{\mathrm{ct}}$ ). The mean level of wakefulness across a 25 hr period for lesioned animals was significantly lower (48\% of $\tau$ and $\left.28.8 \pm 0.9 \mathrm{~min} / \mathrm{hr}_{\mathrm{cl}}\right)$ than intact monkeys $(64 \%$ of $\tau$ and $38.6 \pm 0.7 \mathrm{~min} / \mathrm{hr}_{\mathrm{ct}} ; P<0.001, t$ test).

\section{Effect of SCN lesions on total sleep time and bout lengths}

SCN lesions not only abolished circadian rhythms, but also influenced sleep-stage ratios and increased total sleep time. The percentage distribution of arousal states and total sleep times calculated for individual SCNx monkeys and for the SCNx group is shown in Table 2. On average, SCNx monkeys slept $4.0 \mathrm{hr}$ more per circadian equivalent day length ( $25 \mathrm{hr}$ real time) than intact monkeys $(P<0.01, t$ test $)$. Increased sleep time in SCNx monkeys consisted of SWS 1 , and there was a reciprocal decrease in wakcfulness. SWSl occupied $11.1 \mathrm{hr}$ per $25 \mathrm{hr}$ in SCNx monkeys, which was an average of $3.8 \mathrm{hr}$ per circadian day greater than in intact control animals. Total amounts of SWS2 $(67 \mathrm{~min} / 25 \mathrm{hr}$ ) and REM (46 min/25 hr) observed in SCNx monkeys did not differ significantly from control animals. Arousal state percentages in SCNx monkeys calculated as the mean fraction of total sleep time were SWS1, 85.3\%; SWS2, 8.7\%; and REM, $6.0 \%$.

SCNx monkeys showed markedly reduced wake consolidation as measured by wakefulness bout lengths when compared with intact monkeys during their subjective day [repeated-measures ANOVA between subjects effects, $F(1,8)=1056.5, P<$ 0.0001 ; repeated-measures ANOVA within subjects effects for group by time interaction, $F(1,56)=254.7, P<0.0001]$. The durations of the longest wake and sleep (SWS1, SWS2, and REM) bout lengths within a moving window were calculated and averaged as a function of timc of day for intact and SCNX monkeys (see Materials and Methods) and are shown in Figure 7. Intact monkeys exhibited late subjective day [circadian time (CT) 6-18] wake bout lengths ( $223 \pm 10 \mathrm{~min}$ ) that averaged 15 times greater than those in SCNx monkeys $(15 \pm 6 \mathrm{~min} ; P<$ 0.0001 , two-tailed $t$ test). In contrast, late subjective night (CT 18-21) wake bout lengths averaged only a few minutes less in intact monkeys $(7.9 \pm 2.4 \mathrm{~min})$ than in SCNx monkeys (11.6 $\pm 2.0 \mathrm{~min})$. These differences were not significantly different (REGW multiple $F$ test, $\alpha=0.05$, df $=8$, Critical $F=5.32$ ). Sleep bout lengths were greatest late in the subjective night (CT $18-24)$ in intact monkeys (mean, $25.0 \pm 2.0 \mathrm{~min}$ ) and averaged about 10 min greater than the mean sleep bout lengths observed in SCNx monkeys at corresponding times. It should be noted, however, that individual monkeys exhibited nonstationary ultradian (0.5-6.0 hr period) variations in wake and sleep bout
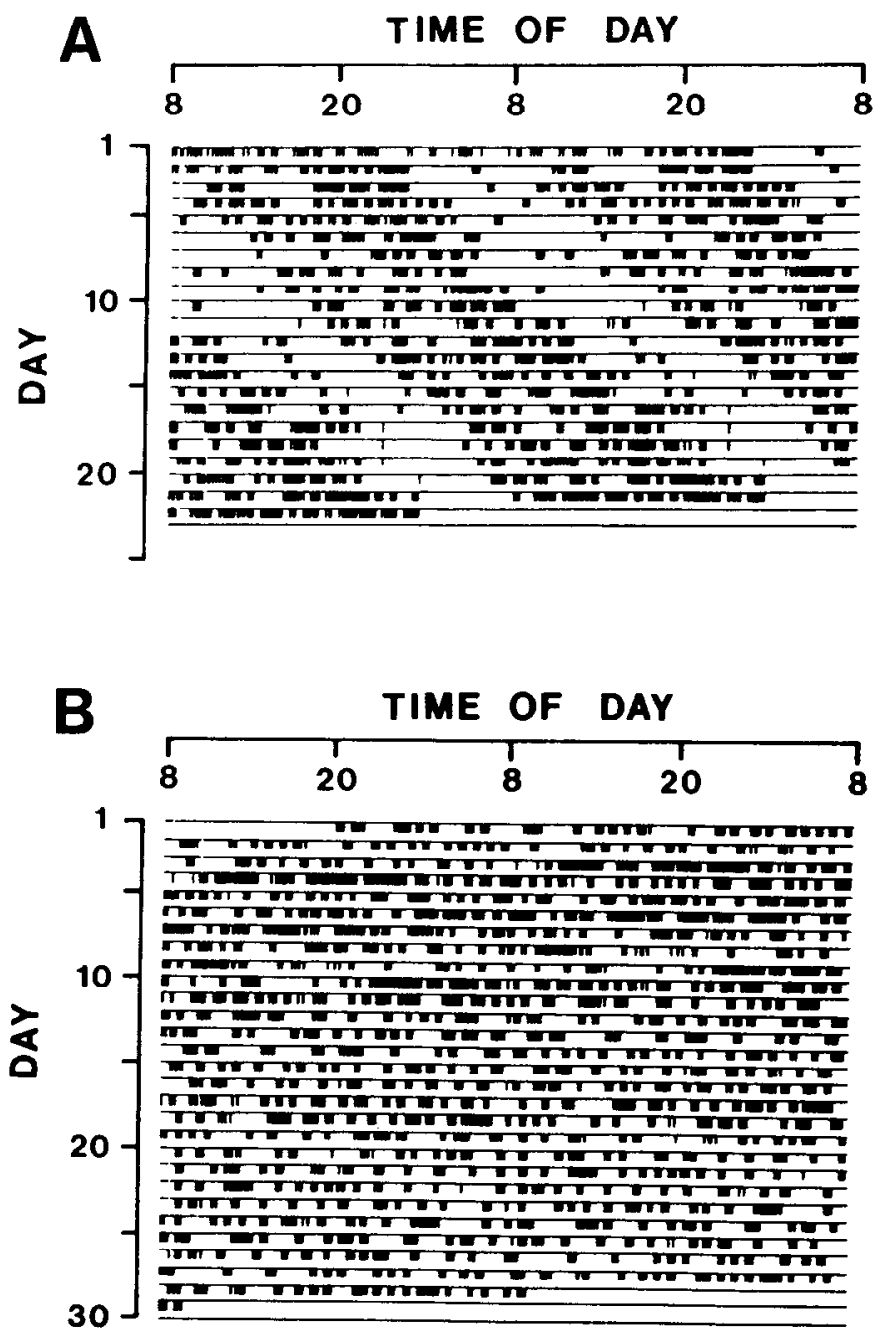

Figure 2. Digital raster plots of drinking obtained from an intact $(A)$ and SCNx $(B)$ squirrel monkey. SCN lesions permanently abolished circadian rhythms in drinking and all other monitored variables. The density of vertical tick marks in the plot are directly proportional to drinking frequency. Data are double plotted to enhance visualization of circadian rhythms. SCNx data shown were obtained 4 months postlesion from monkey $\mathrm{E}$, listed in Table 2.

lengths that are not evident in Figure 7 due to waveform averaging. This was most notable in SCNx monkeys that showed highly variable individual wake bout lengths (15-60 min) and individual sleep bout lengths (20-50 min). When the single longest wake bout length was averaged for intact and SCNx mon-

Table 2. Arousal state distribution in SCNx monkeys

\begin{tabular}{llllll} 
Monkey & \multicolumn{2}{l}{ Mean \pm SD $\%$ state $/ 25 \mathrm{hr}$} & \multicolumn{1}{l}{$\begin{array}{l}\text { TST } / 25 \mathrm{hr} \\
\text { (hr) }\end{array}$} \\
\cline { 2 - 5 } & Awake & SWS1 & SWS2 & REM & 13.6 \\
A & $45.5 \pm 4.8$ & $47.9 \pm 4.3$ & $3.7 \pm 1.0$ & $2.9 \pm 0.2$ & 12.4 \\
B & $50.5 \pm 3.4$ & $42.3 \pm 4.5$ & $4.6 \pm 0.8$ & $2.6 \pm 0.5$ & 13.9 \\
C & $44.2 \pm 2.0$ & $47.3 \pm 2.8$ & $4.9 \pm 1.8$ & $3.6 \pm 0.6$ & 12.0 \\
D & $51.9 \pm 4.1$ & $41.3 \pm 5.2$ & $3.9 \pm 0.9$ & $2.9 \pm 0.4$ & 12.9 \\
E & $48.3 \pm 3.1$ & $42.8 \pm 2.8$ & $5.5 \pm 0.3$ & $3.4 \pm 0.3$ & $13.0 \pm 0.4$ \\
Group \pm SEM & $48.1 \pm 1.5$ & $44.3 \pm 1.4$ & $4.5 \pm 0.3$ & $3.1 \pm 0.2$ &
\end{tabular}




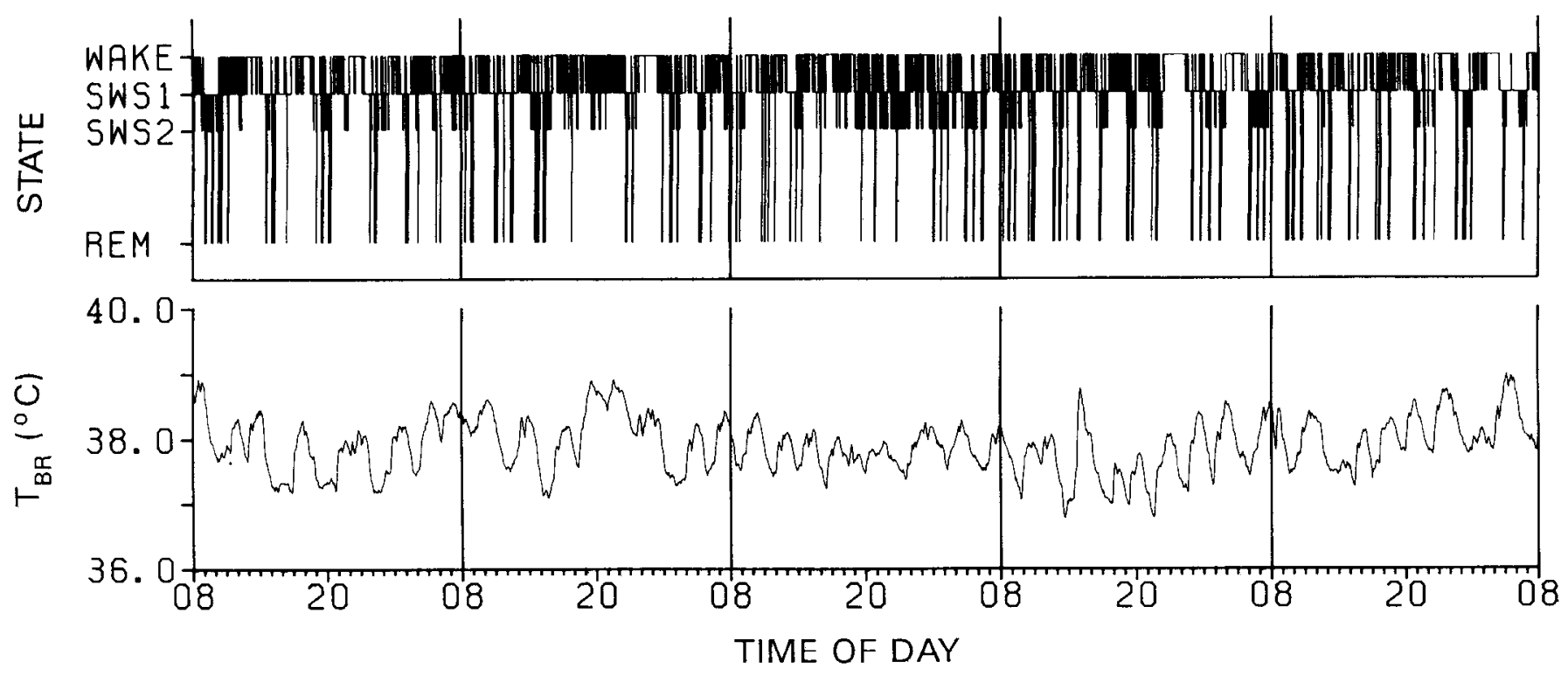

Figure 3. Arousal state and $T_{\mathrm{BR}}$ rhythms monitored from an SCNx squirrel monkey undisturbed in LL. Note that circadian rhythms were absent in each variable, but uitradian variations persisted and were particularly evident in the $T_{\mathrm{BR}}$ data (compare with Fig. 1). Data shown were obtained from monkey A, listed in Table 2.

keys (462 min and $51 \mathrm{~min}$, respectively), and the single longest sleep bout length was similarly averaged (56 min and $52 \mathrm{~min}$ in intact and SCNx monkeys, respectively), it was still clearly evident that SCN lesions resulted in proportionately greater changes in subjective day waking than subjective night sleep or wake.

Sleep fragmentation analysis was performed using a phasespecific bout count analysis technique (see Materials and Methods). Figure 8 shows the mean count of wake and sleep bouts as a function of circadian time for intact and SCNx monkeys. In contrast to intact monkeys, SCNx animals showed no circadian fluctuation in wake or sleep bout counts. During the subjective day, bout counts in intact monkeys were significantly lower than SCNx monkeys due to sustained wake consolidation.
Repeated-measures ANOVA confirmed significant time by group interactions for wake bout counts $[F(1,56)=15.72, P<0.0001]$ and sleep bout counts $[F(1,56)=12.74, P<0.0001]$. During the first half of the subjective night (CT 12-18), both wake and sleep bout counts were statistically indistinguishable between intact and SCNx monkeys (REGW multiple $F$ test, $\alpha=0.05$, $\mathrm{df}=8$, Critical $F=5.32$ ). Both wake and sleep bout counts decreased in intact monkeys during the second half of the subjective night.

\section{Histological verification}

Radio-frequency lesions resulted in complete destruction of the $\mathrm{SCN}$ in all five squirrel monkeys. Figure 9 shows representative histological sections of the anterior hypothalamus from an intact
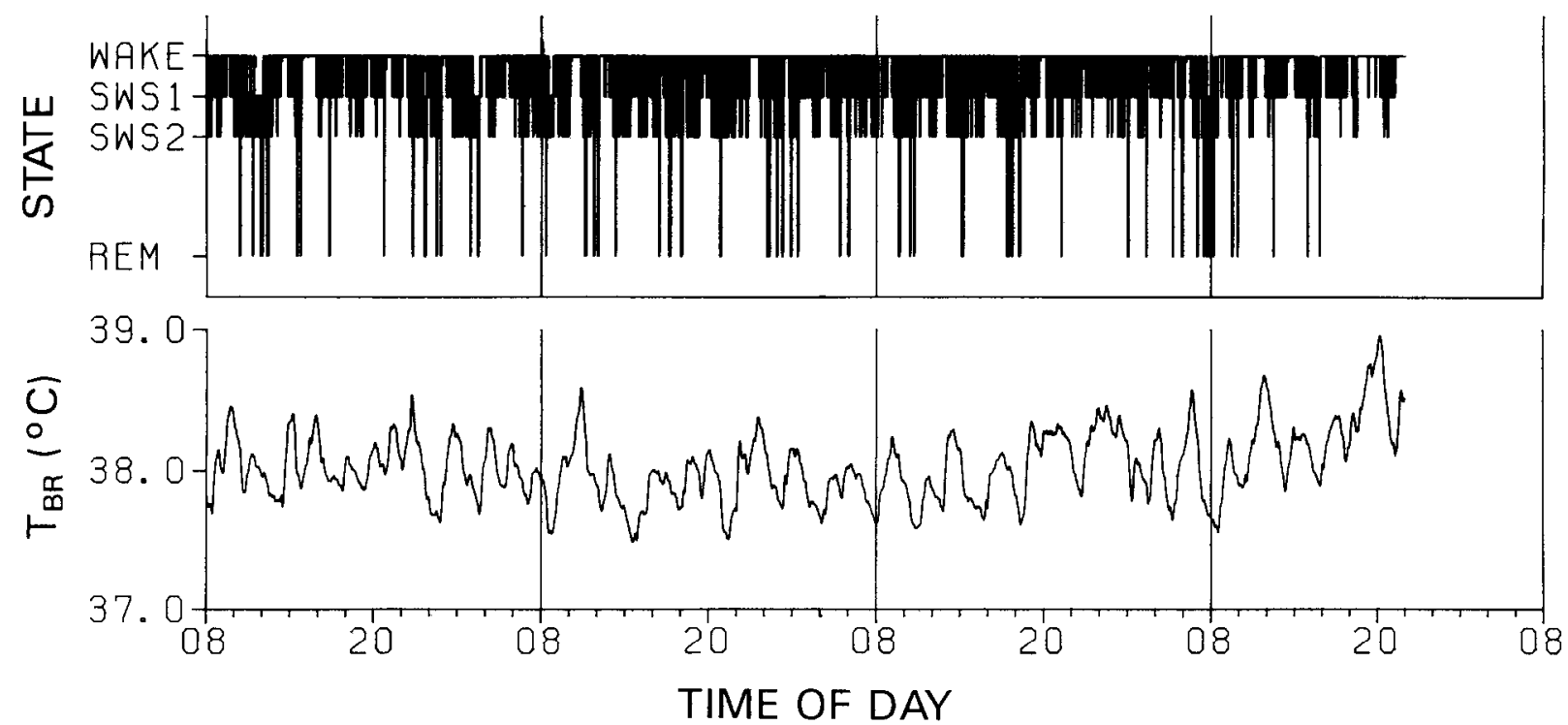

Figure 4. Arousal state and $T_{\mathrm{BR}}$ monitored from an SCNx squirrel monkey exhibiting low-amplitude ultradian rhythms. The amplitude of ultradian variations in arousal state and $T_{\mathrm{BR}}$ varied between animals (compare with Fig. 3). This monkey showed episodic $0.3-0.5^{\circ} \mathrm{C}$ variations in $T_{\mathrm{BR}}$ that corresponded with alternations between waking and sleeping. Data were collected from monkey $\mathrm{D}$, listed in Table 2 , while housed under LL conditions. 
A.) INTACT

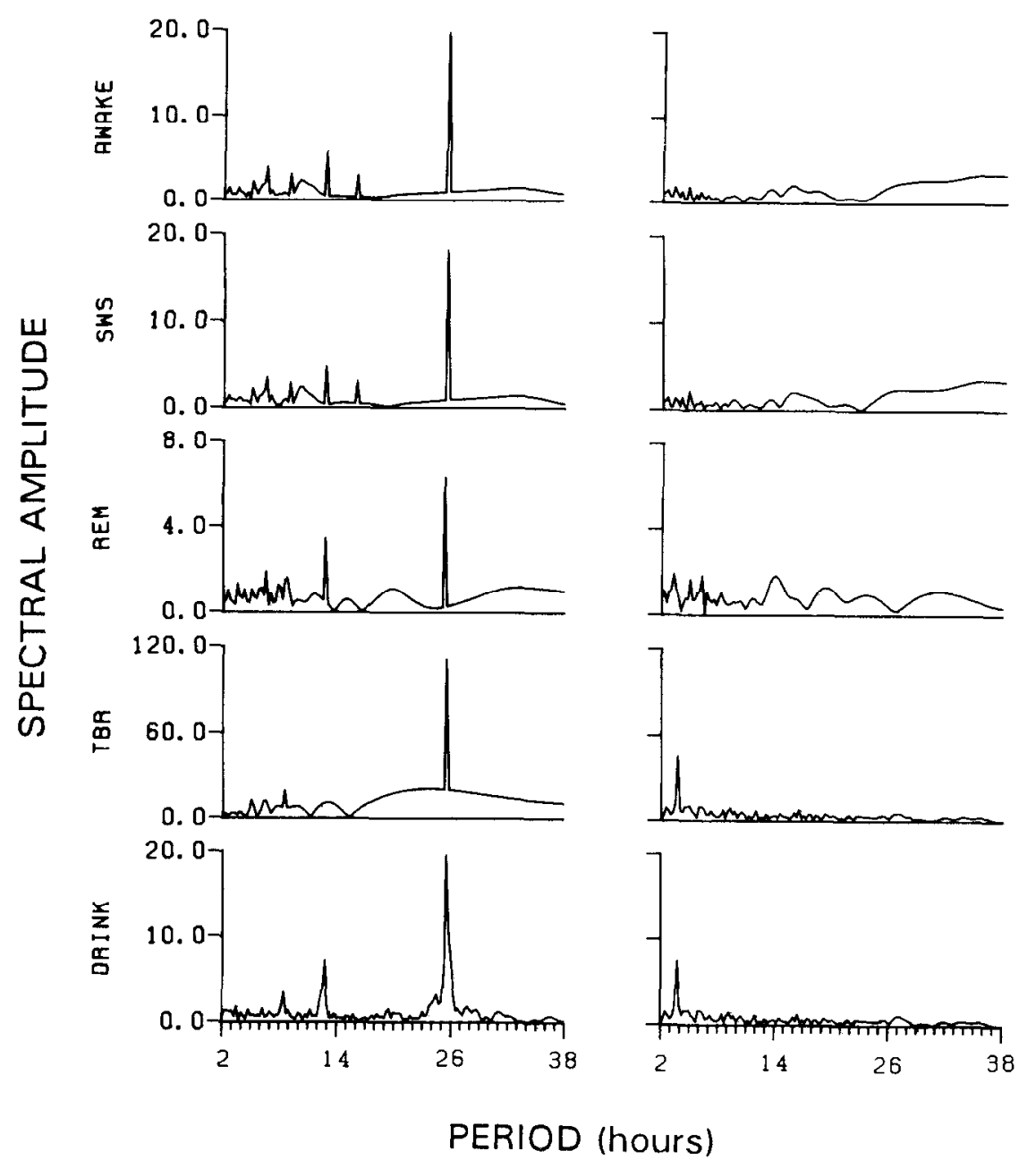

\section{B.) SCNX}

Figure 5. Periodicity analysis of arousal states [awake, SWS1 + SWS2 $(S W S)$, and REM], $T_{\mathrm{BR}}$, and drinking frequency in a sham control $(A)$ and an SCNx $(B)$ squirrel monkey. Peaks in the spectral amplitude indicate rhythm periods with greater relative power. All intact monkeys demonstrated signifcant circadian rhythms $(P<0.05)$ based on parametric (Rummel et al., 1974; shown here) and nonparametric techniques (Sokolove and Bushell, 1978; not shown). No significant circadian component was identified in any variables of lesioned monkeys. control and SCNx monkey. Adjacent histological sections stained for Nissl and Cytox) are shown for comparison. Projection drawings of Cytox-stained sections illustrate the extent of the lesions (Fig. 10). Minor damage may exist in Nissl-stained sections immediately adjacent to the anteriormost and posteriormost Cytox sections shown, but could not be definitively assessed. Damage peripheral to the SCN was limited to approximately $5-10 \%$ of the paraventricular nuclei (PVN), and less than $5 \%$ of the medial preoptic area and medial hypothalamic area. In no case were the supraoptic nuclei damaged. Minor damage to the optic chiasm was found inferior to the SCN in each of the monkeys; however, there was no behavioral indication of visual impairment.

\section{Discussion}

The circadian control of sleep-wakefulness

The present study shows that the primate SCN are not only csscntial for circadian timing, but also influence the regulation of daily total wake and sleep times. To our knowledge, this is the first demonstration of SCN lesion effects on sleep-wakefulness in any primate species, and the first evidence that SCN lesions can affect sleep-wake architecture and markedly change total sleep time. Of the many SCN lesion studies in mammals (for review, see Rusak, 1977; Rusak and Zucker, 1979; Moore,

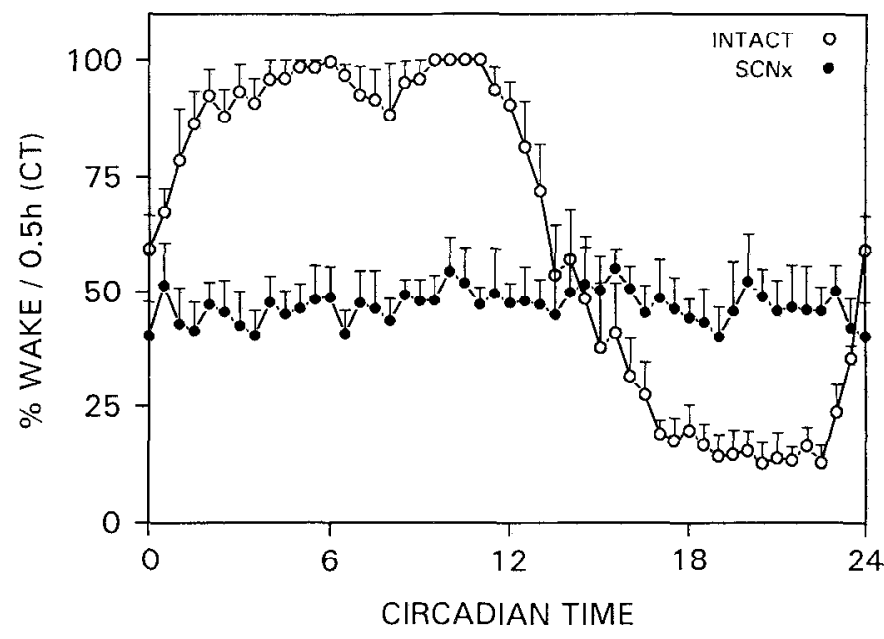

Figure 6. Mean wakefulness waveforms for intact and SCNx squirrel monkeys. On average, SCNX monkeys were awake approximately $48 \%$ of the time, at any time of day. Note also the asymmetry in the wakefulness waveform, reflecting a larger proportion of the circadian day spent awake than asleep in intact monkeys. Time of day is plotted in circadian time, where $24 \mathrm{hr}_{\mathrm{ct}}$ is one circadian period length (see Materials and Methods). Data are plotted as population mean $\pm \mathrm{SEM} ; N=5$ in both groups. 
Figure 7. Temporal variation in the longest wake and sleep bout lengths observed in intact and SCNx monkeys. Temporal profiles were computed using the phase-specific bout length analysis technique (see Materials and Methods). Note the reduction in wake bout lengths during what would otherwise be the subjective day (CT 0-12) in the SCNx population. Data are shown as population mean (solid lines) \pm SEM (broken lines); $N=5$ in each group.
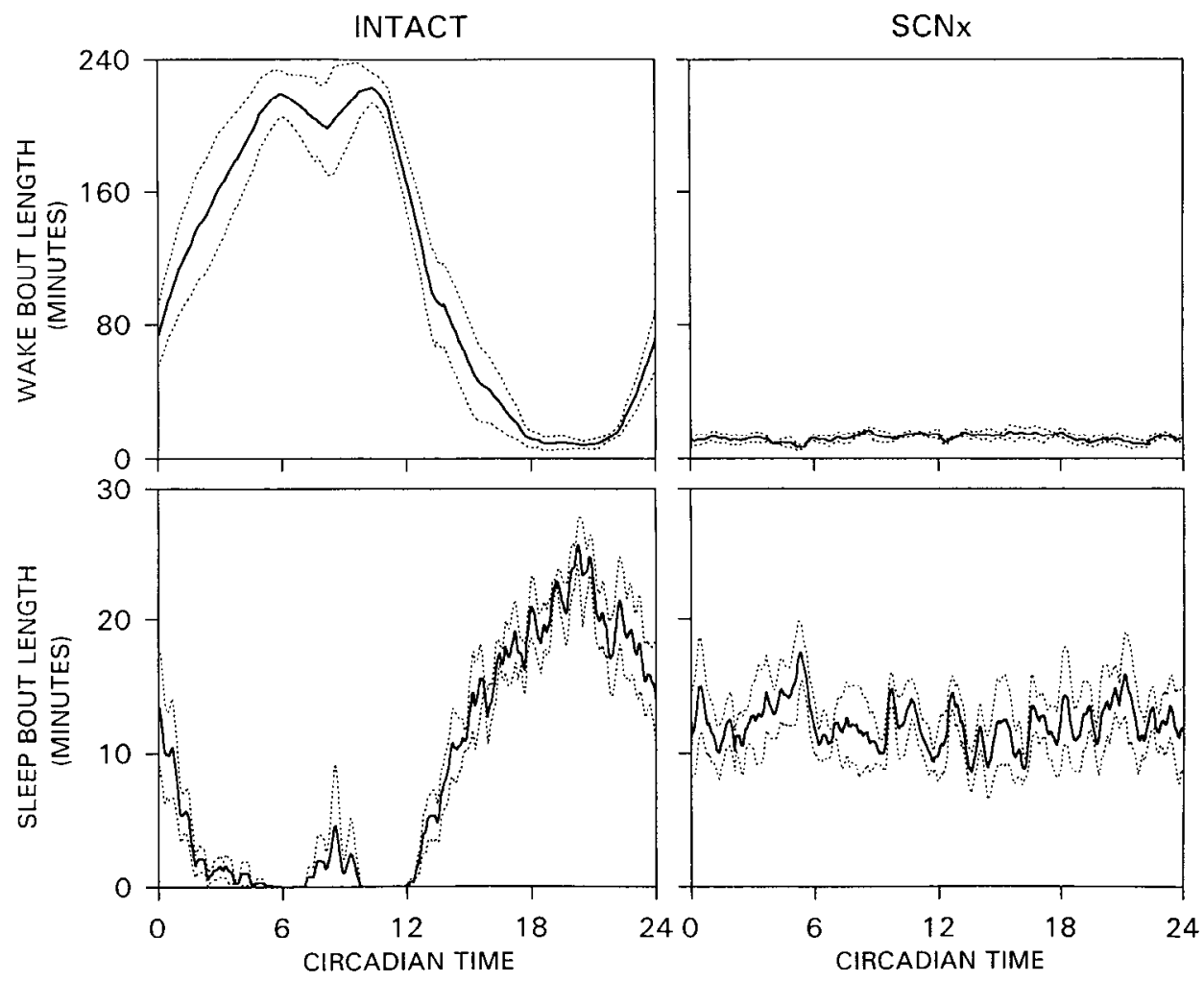

1983; Meijer and Rietveld, 1989; Mistlberger and Rusak, 1989), few have assessed sleep-wake in constant conditions or contrasted sleep patterns with other physiological variables. The elimination of both sleep-wake and cortical $T_{\mathrm{BR}}$ circadian rhythms has been demonstrated in SCNx rats studied in LL
(Eastman et al., 1984). However, unlike the squirrel monkey, which exhibits robust circadian rhythms for years when housed in LL (Richter, 1968), intact rats can exhibit spontaneous degeneration of circadian rhythms in LL (Honma and Hiroshige, 1978) that complicate data interpretation. Sato and Kawamura
Figure 8. Temporal variation in the number of wake and sleep bouts in intact and SCNx monkeys. That fewer wake or sleep bouts were observed during the subjective day (approximately CT 0-12) in intact monkeys reflects long and consolidated bouts of waking (see Fig. 7) with little intervening sleep. Note that bout counts during subjective night phases (approximately CT 12-24) were similar in intact and $\mathrm{SCNx}$ monkeys. Greater wake and sleep bout counts in SCNx monkeys, and during the subjective night in intact monkeys, reflect more frequent transitions between wake and sleep. Data arc plotted as population mean (solid lines) \pm SEM (broken lines); $N=5$ in each group.
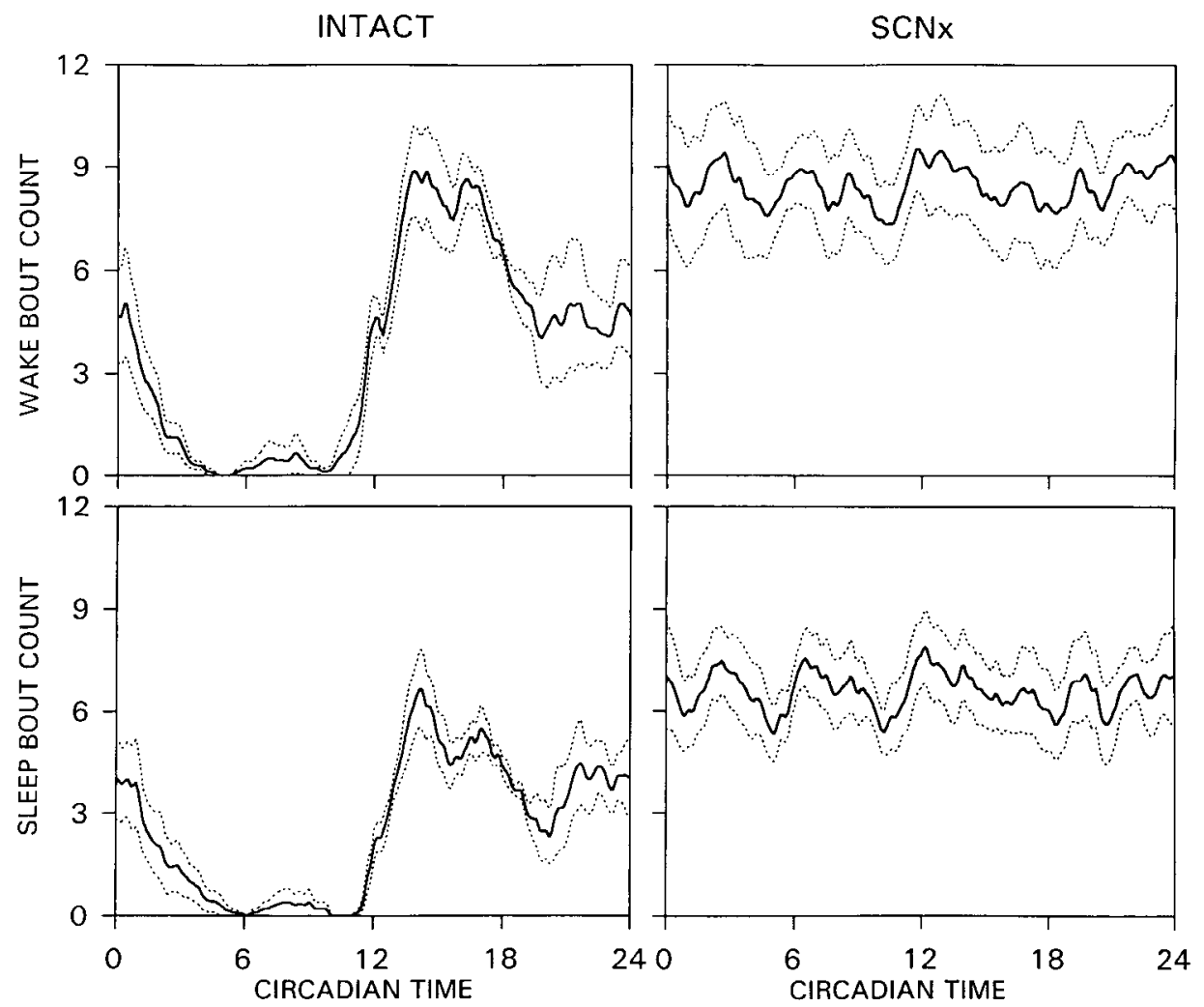


\section{A. INTACT}
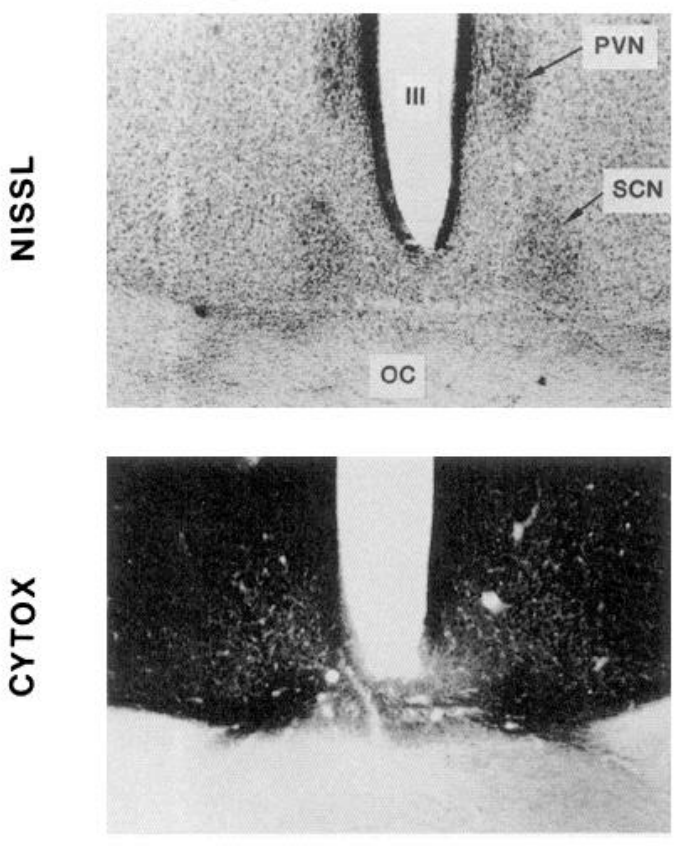

$1 \mathrm{~mm}$

\section{B. LESION}
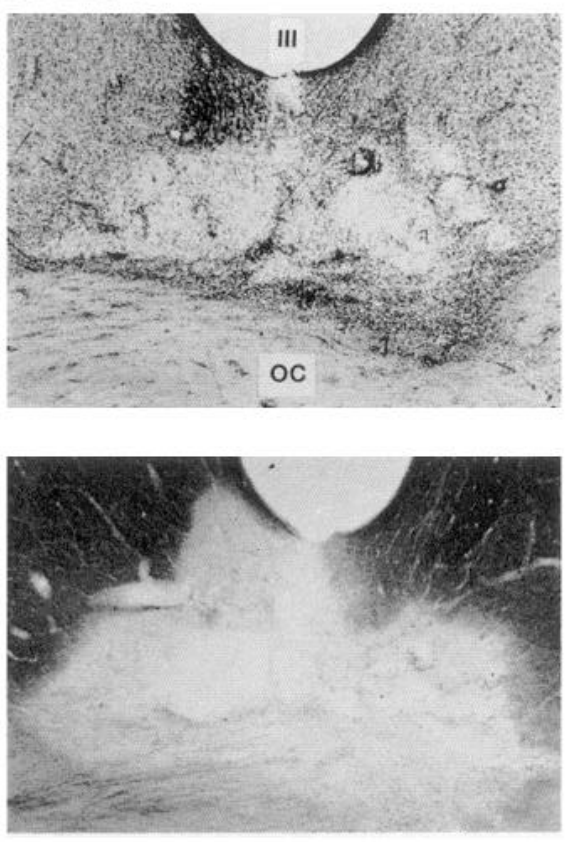

Figure 9. Coronal histological sections through the $\mathrm{SCN}$ of intact $(A)$ and SCNx $(B)$ squirrel monkeys. Adjacent $40 \mu \mathrm{m}$ sections stained for Nissl substance (top) and Cytox activity (bottom) are shown. Note how Cytox staining helps to delimit the extent of the lesion, which totally destroyed the SCN. $O C$, optic chiasm; III, third ventricle. Lesioned animal shown is monkey $\mathrm{A}$, as listed in Table 2, and also shown in Figure 10 . (1984b) found that SCN lesions eliminated circadian rhythms of wake, slow wave sleep, and paradoxical sleep in a diurnal rodent, the Siberian chipmunk. Representative sleep-wake data from one animal in their report suggest that SCN lesions may have increased sleep time during the subjective day, but there was insufficient information to contrast daily sleep amounts in intact and SCNx animals.

In sharp contrast to the 3-4 hr sleep increase in SCNx monkeys, nocturnal rodent studies have revealed little or no change in total sleep time following SCN lesions (Mistlberger et al., 1983). Interspecific variation in normal baseline sleep-wake patterns may be an important determinant of these differences. Unlike the squirrel monkey, which typically showed bouts of uninterrupted wakefulness lasting several hours during the active portion of the circadian day, waking during the activity phase of the rat is normally much more fragmented. Intact rats sleep $30-40 \%$ of the subjective night (activity phase) in constant conditions and show 100 (or more) transitions between sleep and wake throughout the circadian cycle (Borbely and Neuhaus, 1979; Mistlberger et al., 1983). Consequently, rats have much shorter wake bout lengths than squirrel monkeys. Even though SCN lesions completely fragment sleep-wake patterns in both species, the effect on total sleep time may be more dramatic in primates because of the consolidated nature of their sleep-wakefulness.

The large ratio of activity to rest $(\alpha: \rho)$ in monkeys maintained in LL may also be an important factor when assessing changes in sleep time following SCN lesions. Aschoff(1960) showed that $\alpha: \rho$ ratios are systematically influenced by light intensity. In a diurnal primate, the daily duration of activity $(\alpha)$ and endogenous rhythm period $(\tau)$ increases with light intensity (Tokura and Aschoff, 1978; Fuller and Edgar, 1986). In rodents, $\alpha$ is inversely correlated with light intensity. Thus, rats maintained in LL could conceivably show a small decrease in sleep time after SCN lesions, whereas the opposite would be predicted for
SCNx rats in constant darkness. If such were the case, then rodents, like squirrel monkeys, may also evince sleep regulation through clock-dependent mechanisms controlling waking if housed under different lighting conditions (i.e., darkness) than have been used to date.

When interpreting the effects of SCN lesions in sleep-wakefulness, it is important to consider the potential consequences of collateral hypothalamic damage. For example, damage to the anterior hypothalamus, basal forebrain, and medial preoptic area (adjacent to the SCN) decreases sleep time in cats (McGinty and Sterman, 1968) and rats (Nauta, 1946; Szymusiak and Satinoff, 1984)-effects diametrically opposite to those produced by $\mathrm{SCN}$ lesions in squirrel monkeys. Although one may argue interspecific differences, it seems unlikely that the increased total sleep time in SCNx monkeys was a product of collateral damage. Speculatively, however, sufficient collateral damage anterior to the SCN could potentially counter the sleep-increasing effects of discrete SCN lesions, eventuating no net change in total sleep time (as has been reported in rats). Recent studies of sleep architecture in rats with incomplete SCN lesions or very small but complete SCN lesions show 30-50 min increases in total sleep time (D. M. Edgar, unpublished observation), consistent with the view that small lesions of the SCN are necessary to increase sleep time in mammals.

There is some evidence that SCN lesions can alter the levels of motor activity in rodents (Johnson et al., 1988), raising the possibility that sleep changes could be secondary to reduced motor function. In mice, motor activity is an important determinant of sleep-wake cycle consolidation (Welsh et al., 1988, Edgar et al., 1991a). It is not clear, however, whether this phenomenon can be generalized to other species (Borbely and Hanagasioglu, 1982). Although sleep can be influenced by restraint in primates (Bert et al., 1975), there do not appear to be significant differences between the circadian rhythms of restrained and freely behaving squirrel monkeys (Moore-Ede et al., 1982). 

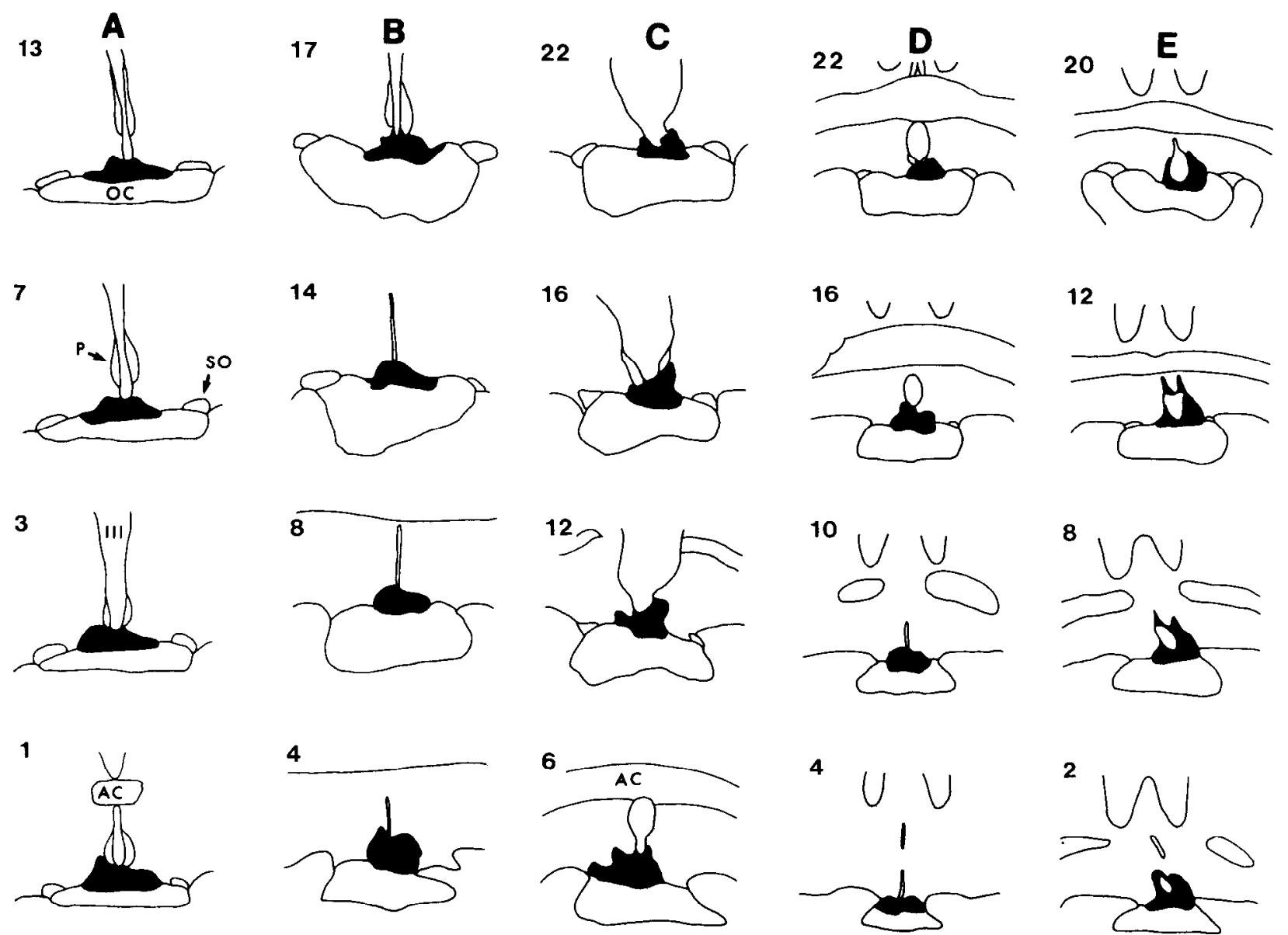

$2.0 \mathrm{~mm}$

Figure 10. Projection drawings of coronal sections through the anterior hypothalamus of the five SCNx squirrel monkeys $(A-E)$. Drawings were based on Cytox-stained sections. Anterior sections are on the bottom; posterior sections are on the top. Section number is shown in the upper left corner of each drawing for reference. $A C$, anterior commissure; $O C$, optic chiasm; $P, P V N ; S O$, supraoptic nucleus; $I I I$, third ventricle.

In the present study, monkeys were not restrained; however, we did not directly measure motor activity within the cage. Nonetheless, there was no difference in mean $T_{\mathrm{BR}}$, food and water consumption, or closed-circuit video observations of exploratory behavior and play, which would have indicated that, aside from increased sleep time, a fundamental change in the activity level of SCNx monkeys occurred.

The increase in sleep time and decrease in subjective day wake bout lengths produced by SCN lesions offer possible new insights into the circadian control of sleep-wakefulness. The 15-fold reduction in wake bout lengths in SCNx monkeys and the similarity in subjective night wake bout lengths and bout counts in intact and SCNx monkeys strongly suggest that the SCN promotes wakefulness during the subjective day. There appears to be much less circadian control imposed over arousal states during the subjective night since sleep and wake bout counts (assessments of sleep-wake fragmentation) and sleep and wake bout lengths in intact monkeys more closely approximated that of SCNx monkeys at those times of day.

Presently, it is not clear whether the greater length of sleep bouts in intact monkeys, when compared to sleep bout lengths in SCNx monkeys, is the result of SCN influences on compen- satory sleep mechanisms (e.g., a homeostatic sleep-promoting process), reflects recovery sleep in response to waking initiated and/or maintained by the SCN, or both. SCNx rats exhibit recovery sleep following sleep deprivation (Mistlberger et al., 1983; Tobler et al., 1983), suggesting that a homeostatic sleeppromoting process can function independent of circadian control. Other homeostatic functions (e.g., thermoregulation) remain intact in SCNx animals as well (Edgar, 1986). If homeostasis remains intact, why then would total sleep time per day increase in SCNx monkeys? One possible explanation is that the amount of compensatory sleep necessary for homeostatic recovery is less than that displaced by prior wakefulness. If the gain of the sleep homeostatic process is an exponential function related to prior sleep loss (cf. Borbely et al., 1989), then the reduction in wake consolidation (and reduction in accumulated sleep loss) in SCNx monkeys would result in a disproportionate decrease in compensatory drive responsible for subjective night sleep consolidation. Alternatively, sleep may be less efficient in SCNx monkeys due to fragmentation. The relation of fragmentation to the restorative value of sleep is well documented in humans (Bonnet, 1987; Levine et al., 1987). Exactly how sleep fragmentation diminishes the value of sleep is unclear, but may 
be due to the amount of EEG delta sleep accumulated per hour of total sleep. EEG delta power appears to be a good marker of homeostatic sleep drive (Borbely and Neuhaus, 1979; Borbely et al., 1981) and is related to the duration of prior waking (Tobler and Borbely, 1986; Dijk et al., 1987, 1990). Consistent with this interpretation is the finding that SWS2 and REM time were essentially unchanged in SCNx monkeys. In fact, the increase in total sleep time was almost exclusively due to increased SWS1, which is characterized by low (and perhaps inefficient) levels of delta EEG. Thus, the SCN appear to have two important benefits affecting sleep and wakefulness in the squirrel monkey: (1) enhanced sleep-wake cycle consolidation through the promotion of subjective day wakefulness and (2) increased nocturnal sleep efficiency as a consequence of consolidating waking.

\section{Opponent processes in sleep-wake regulation}

Historically, regulation of the circadian sleep-wake cycle has been characterized by the interaction of physiological systems. Such concepts have offered logical constructs that aid the search for mechanisms at the cellular level. Conventional views posit that the circadian rhythm of sleep-wakefulness reflects an oscillating process that is gated by thresholds (Aschoff et al., 1971). To these ends, Borbely, Daan, and colleagues have offered convincing evidence that the sleep-wake cycle is the product of systematic interactions between the circadian timing system and a sleep homeostatic process (Borbely, 1982). As developed and mathematically characterized in their "two-process model" of sleep-wake regulation, a homeostatically regulated sleep process is gated by circadian oscillations in thresholds that determine wake and sleep onsets (Daan et al., 1984; Borbely et al., 1989). Despite the utility of this nodel in describing and predicting aspects of animal and human sleep timing (Strogatz, 1986), its conceptual framework defining circadian control has embodied and generalized the observation in nocturnal rodents that total sleep time is unaffected by SCN lesions. This generalization is not consistent with the present observation in diurnal primates, and may have confounded the development of more specific insights into the modulation of arousal states by the SCN.

The selective facilitation of wakefulness by the primate $\mathrm{SCN}$ clarifies the functional role of the circadian timing system and implicates an alternative to threshold-based notions of sleepwake cycle regulation. Inspired by the work of Borbely and colleagues, but based on the primate data presented here, an alternative conceptual model considers sleep-wake regulation in probabilistic terms such that the $\mathrm{SCN}$ promote wakefulness and thus oppose the sleep homeostatic process. In this view, physiological sleep tendency (or its converse, alertness) is determined by the sum of these two opponent processes. This "opponent process" model implies that circadian oscillations in sleep drive are indirectly caused by SCN-dependent mechanisms that impose prolonged intervals of waking (sleep loss) each circadian day.

An opponent process model of sleep-wake regulation is consistent with increased sleep time and shortened wake bout lengths in SCNx monkeys and with sleep-wake timing in humans as well. The circadian timing of spontaneous waking in free-running humans is generally more predictable than sleep onsets. Indeed, the duration of sleep is a function of the circadian phase of sleep onset because of the precise timing of waking by the circadian system (Czeisler et al., 1980). If the circadian timing system were not responsible for promoting waking, it would be difficult to understand why jet lag and shift work would result in waking at inappropriate times, even when subjects have experienced considerable sleep loss (Seidel et al., 1986). In addition, physiological sleep tendency, as assessed by the multiple sleep latency test, shows circadian variations in sleep-deprived subjects (Carskadon and Dement, 1979, 1982), consistent with the concept that a "circadian clock"-regulated mechanism opposes homeostatic sleep drive at specific times of day (Mistlberger et al., 1983; Edgar, 1986; Strogatz et al., 1986, 1987; Borbely et al., 1989).

The opponent process model further implies that the lack of circadian-imposed wakefulness after an SCN lesion will enable the animal to sleep at any time of day. Like the two-process model, the sleep homeostatic process is viewed as a relaxation oscillation or "hourglass" reflecting the duration of prior wakefulness (Dijk et al., 1987). Therefore, in a constant environment, the SCNx animal will sleep as soon as minimal sleep tendency has built up. This state may be called "chronic sleep satiation" since the SCNx animal does not accumulate appreciable sleep loss during what would otherwise be the subjective day. Two lines of evidence suggest such is the case. First, SCNx monkeys (and SCNx rats; Edgar, unpublished observations) have much shorter wake bouts and appeared to be more easily aroused than intact controls. Second, SCNx rats show markedly attenuated sleep responses to benzodiazepine sedative hypnotics (Edgar et al., 1991c). The soporific effects of benzodiazepines (Edgar et al., 1991b) and ethanol (Roehrs et al., 1989) vary as a function of prior wakefulness, and benzodiazepine soporific effects can be restored in SCNx rats deprived of sleep for $6 \mathrm{hr}$ prior to treatment (Edgar et al., 1990; Trachsel et al., 1992).

Opponent process regulation can also be extended to account for the temporal dynamics of sleep tendency in both monkeys and humans. Sleep propensity apparently increases as the night progresses, even as "deep" slow-wave sleep-a product of homeostatic sleep drive according to the two-process model-diminishes exponentially. In the intact squirrel monkey, sleep bout length and sleep time per hour increase throughout the night, but SWS2 subsides (Edgar, 1986). In humans, latency to return to sleep shortens during the night (Richardson et al., 1978, 1982), even though "deep" slow wave sleep is diminishing. The opponent process model could explain this phenomenon by assuming an SCN wake-promoting signal; sleep continuity as the night progresses is assured by the diminution of the SCN signal, plus residual homeostatic sleep drive.

The concept of opponent regulatory processes diverges from the notion that sleep and wake are binary expressions of a unitary physiological phenomenon. Instead, this model reflects growing evidence that sleep-wake manifestation is the product of complex interactions between multiple physiological systems; diametrically opposite functions of an SCN-dependent activating process and a homeostatic sleep-promoting process constitute the simplest derivation of opponent process regulation. That only one central circadian pacemaker is needed for opponent process regulation further contributes to its simplicity and may help bridge the circadian sleep-wake phenomenology in humans and animals. A forthcoming mathematical description of the opponent process model should aid in testing its predictive value in animals as well as human sleep-wake pathophysiology.

\section{Anatomical considerations}

Lesion studies constitute the largest body of data implicating the SCN in circadian timekeeping, but not without controversy. 
Mixed reports of persisting rhythmicity in one or more variables after SCN lesions (Stephan et al., 1979; Fuller et al., 1981; Reppert et al., 1981; Satinoff and Prosser, 1988; Kittrell, 1991) have been viewed as evidence of a second circadian pacemaker governing such variables as body temperature, REM sleep, and certain endocrine rhythms (cf., Moore-Ede et al., 1981; Kronauer et al., 1982). In some cases, disparity between lesion studies may be attributed to differences in methodological techniques, sex, species, or the use (and abuse) of statistical techniques to evaluate the presence or absence of rhythmicity (for review, see Kittrell, 1991). Taking a conservative view, loss of brain temperature, drinking, and sleep-wake circadian rhythms (including component sleep stages) in unrestrained SCNx monkeys shows that the neural structures essential to the circadian timing of these variables must be located in close proximity to each other (e.g., within or immediately adjacent to the SCN). Less conservatively, it may be argued that these data obviate the notion of separate pacemakers underlying sleep-wake and body temperature circadian rhythms. Both views are also supported by recent evidence that fetal $\mathrm{SCN}$ transplants can restore circadian rhythmicity to locomotor activity, sleep-wake, and body temperature (Edgar et al., 1992).

Why the present data disagree with the earlier work of Fuller and colleagues in the same species is not yet clear, although limitations in earlier histological validations may be important. Data from one partially SCNx monkey with only $10 \%$ of the posterior SCN remaining in one nuclei (not reported here due to lack of sleep-wake data) revealed persisting low-amplitude $\left(0.2-0.3^{\circ} \mathrm{C}\right) T_{\mathrm{BR}}$ circadian rhythms but no "statistically" resolvable circadian feeding or drinking patterns (Edgar, 1986). This observation and the restoration of circadian rhythms by transplantation of cultured fetal SCN cells (Ralph and Lehman, 1991) lend credence to the notion that relatively little SCN tissue is required to generate circadian rhythms.

Specific SCN efferent pathways, related neurotransmitters, and target structures responsible for the circadian control of arousal states are not known, although the present study may offer an important clue. Von Economo (1931) concluded that a "sleep-regulating center" consisting of antagonistic parts (e.g., distinct wake-promoting and sleep-promoting components) was present within the midbrain and diencephalon. It is now clear that there are many structures and pathways involved in the wake- and sleep-generating systems (for review, see Jones, 1989; Steriade and McCarley, 1990), and that the SCN may differentially affect these systems.

The majority of SCN efferents project dorsocaudally to the subparaventricular zonc, a region between the borders of the SCN and the ventral margin of the PVN (Watts, 1991). However, neurons in both the subparaventricular zone and the SCN project to several regions including the medial preoptic area, posterior hypothalamus, septal nuclei, paraventricular thalamus, basal forebrain, and rostral brainstem (Card et al., 1981; Stephan et al., 1981; Sofroniew and Weindl, 1982; Watts et al., 1987; Jones, 1989; Watts, 1991), all of which are thought to be important in sleep-wake behavior (Hernandez-Peon and Chavez-Ibarra, 1963; Jouvet, 1969; Sterman and Clement, 1974; Obal et al., 1982; Sterman and Shouse, 1985; Steriade, 1988, 1989; Jones, 1989; Steriade and McCarley, 1990).

Given the present evidence that the SCN differentially promotes wakefulness, it is tempting to speculate that circadian control is imparted through neuronal populations that invoke cortical activation. Evidence that rest-activity circadian rhythms persist in animals with midbrain transections (Hanada and Kawamura, 1981) suggests that SCN effector mechanisms act on supramesencephalic brain regions controlling cortical arousal. Consistent with this view, Watts (1991) postulated that the SCN could mediate rhythms in sleep-wakefulness and motivated behaviors by influencing activity of the medial forebrain bundle through projections into the limbic system. The ascending reticular activating system includes a ventral pathway to the basal forebrain and cortex via the ventral thalamus, subthalamus, and posterior hypothalamus (Jones and Yang, 1985; Jones, 1989), the latter of which may be especially important in the manifestation of behavioral wakefulness (Nauta, 1946; Feldman and Waller, 1962). Descending projections of the posterior hypothalamus may also stimulate midbrain reticular neurons involved in the maintenance of the thalamocortical activating process (cf. Steriade and McCarley, 1990). It should be noted, however, that the most powerful drive of all activating supramesencephalic structures is thought to arise in the upper brainstem reticular core (Steriade and McCarley, 1990), which also receives projections from the SCN (Kucera and Favrod, 1979; Stephan et al., 1981). The network of neuronal interactions in the cortical activation process are complex and involve reciprocal innervation between the mesencephalic reticular nuclei and the various supramesencephalic brain regions involved (cf. Steriade and McCarley, 1990). Thus, additional work will be necessary to establish a direct link between SCN neuronal activity and specific neuronal populations thought to be centers of arousal state control.

\section{Conclusions}

Lesions of the primate $\mathrm{SCN}$ revealed that the circadian system is instrumental in both circadian sleep-wake cycle timing and regulation of total sleep time. In the intact monkey, the circadian system functions to promote wakefulness during the subjective day but does not appear to promote sleep actively during the subjective night. How SCN effector mechanisms achieve this arousal state control is not known. The use of sophisticated neuroanatomical tracing techniques to define the afferent and efferent projections and intrinsic structure of the SCN (cf. Van den Pol, 1991; Watts, 1991) has helped to assuage some concerns about differentiating SCN lesion effects from unavoidable collateral damage. However, caution should be exercised when interpreting the functional role of the $\mathrm{SCN}$ and its primary efferent relays within the subparaventricular zone-especially in comparisons between diurnal and nocturnal species. Both diurnal and nocturnal mammals exhibit peak neural activity within the SCN during the subjective day (Schwartz et al., 1983), but unlike diurnal mammals, the electrical activity of neurons in the peri-SCN region of nocturnal rodents exhibits circadian rhythms that are antiphase with SCN neuronal activity (Kubota et al., 1981; Sato and Kawamura, 1984a). Thus, cells immediately adjacent to the SCN could define the polarity of SCN effector function underlying diurnal and nocturnal sleep-wake patterns.

Consistent with rodent studies (Mistlberger et al., 1983), the SCN does not appear essential to the mechanisms underlying the homeostatic regulation of SWS2 or the ultradian timing of the REM-non-REM sleep cycle in the squirrel monkey. The latter is thought to be separately controlled by reciprocal interactions between pontine excitatory and inhibitory neuronal populations (Hobson et al., 1975; McCarley and Massaquoi, 1992). Collectively, these findings suggest that the circadian manifes- 
tations of sleep and wake may be the resultant of opponent physiological processes-an SCN-dependent process that promotes wakefulness at specific times of day, and a sleep homeostatic process that increases sleep drive in response to waking imposed either directly or indirectly by the SCN. Regulation through opponent processes is a common strategy in mammals (e.g., separate heat production and heat loss effector mechanisms in thermoregulation, endocrine regulation by releasing and inhibiting factors, etc.). The SCN may, however, differentially modulate various control systems and effector mechanisms that are functionally integrated with arousal state control and collectively determine manifest sleep-wake behavior.

\section{References}

Adams PM, Barratt ES (1974) Nocturnal sleep in squirrel monkeys. Electroencephalogr Clin Neurophysiol 36:201-204.

Aschoff J (1960) Exogenous and endogenous components in circadian rhythms. Cold Spring Harbor Symp Quant Biol 25:1 1-28.

Aschoff J, Gerecke U, Kureck A, Pohl H, Rieger P, von Saint-Paul U, Wever R (1971) Interdependent parameters of circadian activity rhythms in birds and man. In: Biochronometry, Vol 1 (Menaker M, ed), pp 3-29. Washington, DC: National Academy of Sciences.

Bert J, Balzamo E, Chase M, Pegram V (1975) The sleep of the baboon, Papio papio, under natural conditions and in the laboratory. Electroencephalogr Clin Neurophysiol 39:657-662.

Bonnet MH (1987) Sleep restoration as a function of periodic awakening, movement, or electroencephalographic change. Sleep 10:364373.

Borbely AA (1982) A two process model of sleep regulation. Hum Neurobiol 1:195-204.

Borbely AA, Hanagasioglu M (1982) Effect of locomotor activity on sleep in the rat. Behav Brain Res 4:359-368.

Borbely AA, Neuhaus HU (1979) Sleep-deprivation: effects on sleep and EEG in the rat. J Comp Physiol 133:71-87.

Borbely AA, Baumann F, Brandeis D, Strauch I, Lehmann D (1981) Sleep deprivation: effect on sleep stages and EEG power density in man. Electroencephalogr Clin Neurophysiol 51:483-493.

Borbely AA, Achermann P, Trachsel L, Tobler I (1989) Sleep initiation and initial sleep intensity: interactions of homeostatic and circadian mechanisms. J Biol Rhythms 4:149-160.

Card JP, Brecha N, Karten HJ, Moore RY (1981) Immunocytochemical localization of vasointestinal peptide containing cells and processes in the suprachiasmatic nucleus of the rat: light and electron microscopic analysis. J Neurosci 1:1289-1303.

Carskadon MA, Dement WC (1979) Effects of total sleep loss on sleep tendency. Percept Motor Skills 48:495-506.

Carskadon MA, Dement WC (1982) Nocturnal determinants of daytime sleepiness. Sleep 5:S73-S81.

Czeisler CA, Weitzman ED, Moore-Ede MC, Zimmerman JC, Knauer RS (1980) Human sleep: its duration and organization depend on its circadian phase. Science 210:1264-1267.

Daan S, Beersma DGM, Borbely AA (1984) Timing of human sleep: recovery process gated by a circadian pacemaker. Am J Physiol 246: R161-R 178 .

Dijk DJ, Beersma DGM, Daan S (1987) EEG power density during nap sleep: reflection of an hourglass measuring the duration of prior wakefulness. J Biol Rhythms 2:207-219.

Dijk DJ, Brunner DP, Beersma DG, Borbely AA (1990) Electrogram power density and slow wave sleep as a function of prior waking and circadian phase. Sleep 13:430-440.

Dinges DF (1989) The influence of the human circadian timekeeping system on sleep. In: Principles and practice of sleep medicine (Kryger MH, Roth T, Dement WC, eds), pp 153-172. Philadelphia: Saunders.

Eastman CI, Mistlberger RE, Rechtschaffen A (1984) Suprachiasmatic nuclei lesions eliminate circadian temperature and sleep rhythms in the rat. Physiol Behav 32:357-368.

Edgar DM (1986) Circadian timekeeping in the squirrel monkey: neural and photic control of sleep, brain temperature and drinking. PhD thesis, University of California, Riverside.

Edgar DM, Trachsel L, Seidel WF, Heller HC, Dement WC (1990) The efficacy of triazolam is restored in sleep-deprived SCN-lesioned rats. Sleep Res 19:352.
Edgar DM, Kilduff TS, Martin CE, Dement WC (1991a) Influence of running wheel activity on free-running sleep/wake and drinking circadian rhythms in mice. Physiol Behav 50:373-378.

Edgar DM, Seidel WF, Dement WC (1991b) Triazolam-induced sleep in the rat: influence of prior sleep, circadian time, and light/dark cycles. Psychopharmacology 105:374-380.

Edgar DM, Seidel WF, Martin CE, Sayeski PP, Dement WC (1991c) Triazolam fails to induce sleep in suprachiasmatic nucleus-lesioned rats. Neurosci Lett 125:125-128.

Edgar DM, Ralph MR, Scidel WF, Lec LK, Dcment WC (1992) Fetal SCN-transplants restore sleep-wake and body temperature circadian rhythms in SCN-lesioned rats. Sleep Res 21:371.

Emmers R, Akert KA (1963) Stereotaxic atlas of the brain of the squirrel monkey (Saimiri sciureus). Madison, WI: University of Wisconsin.

Feldman SM, Waller HJ (1962) Dissociation of electrocortical activation and behavioral arousal. Nature 196:1320-1322.

Fuller CA, Edgar DM (1986) Effects of light intensity on the circadian temperature and feeding rhythms in the squirrel monkey. Physiol Behav 36:687-691.

Fuller CA, Lydic R, Sulzman FM, Albers HE, Tepper B, Moore-Ede MC (1981) Circadian rhythm of body temperature persists after suprachiasmatic lesions in the squirrel monkey. Am J Physiol 241: R385-R391.

Gander PH, Moore-Ede MC (1983) Light-dark masking of circadian temperature and activity rhythms in squirrel monkeys. Am J Physiol 245:R927-R934.

Green DJ, Gillette R (1982) Circadian rhythm of firing rate recorded from single cells in the rat suprachiasmatic slice. Brain Res 245:198200.

Groos GA, Hendriks I (1982) Circadian rhythms in electrical discharge of rat suprachiasmatic neurons recorded in vitro. Neurosci Lett 34 : 283-288.

Hanada Y, Kawamura H (1981) Sleep-waking electrocorticographic rhythms in chronic cerveau isole rats. Physiol Behav 26:725-728.

Hernandez-Peon R, Chavez-Ibarra G (1963) Sleep induced by electrical or chemical stimulation of the forebrain. Electroencephalogr Clin Neurophysiol [Suppl] 24:188-198.

Hobson JA, McCarley RW, Wyzinski PW (1975) Sleep cycle oscillation: reciprocal discharge by two brainstem neuronal groups. Science 189:55-58.

Honma K, Hiroshige T (1978) Endogenous ultradian rhythms in rats exposed to prolonged continuous light. Am J Physiol 235:R250-R256.

Johnson RF, Moore RY, Morin LP (1988) Running wheel activity in hamsters with hypothalamic damage. Physiol Behav 43:755-763.

Jones BE (1989) Basic mechanisms of sleep-wake states. In: Principles and practice of sleep medicine (Kryger MH, Roth T, Dement WC, eds), pp 121-138. Philadelphia: Saunders.

Jones BE, Yang T-Z (1985) The efferent projections from the reticular formation and the locus coeruleus studied by anterograde and retrograde axonal transport in the rat. J Comp Neurol 242:56-92.

Jouvet M (1969) Biogenic amines and the states of sleep. Science 163: $32-41$.

Kittrell EMW (1991) The suprachiasmatic nucleus and temperature rhythms. In: Suprachiasmatic nucleus: the mind's clock (Klein DC, Moore RY, Reppert SM, eds), pp 233-245. New York: Oxford UP.

Kronauer RE, Czeisler CA, Pilato SF, Moore-Ede MC, Weitzman ED (1982) Mathematical model of the human circadian system with two interacting oscillators. Am J Physiol 242:R3-R17.

Kubota A, Inouye ST, Kawamura H (1981) Reversal of multiunit activity within and outside the suprachiasmatic nucleus in the rat. Neurosci Lett 27:303-308.

Kuccra P, Favrod P (1979) Suprachiasmatic nucleus projections to mesencephalic grey in the woodmouse (Apodemus sylvaticus $L$.). Neuroscience 4:1705-1715.

Levine B, Roehrs T, Stepanski E, Zorick F, Roth T (1987) Fragmenting sleep diminishes its recuperative value. Sleep 10:590-599.

McCarley RW, Massaquoi SG (1992) Neurobiological structure of the revised limit cycle reciprocal interaction model of REM cycle control. J Sleep Res 1:132-137.

McGinty DJ, Sterman MB (1968) Sleep suppression after basal forcbrain lesions in the cat. Science 160:1253-1255.

McNew JJ, Burson RC, Hoshizaki T, Adey WR (1972) Sleep-wake cycle of an unrestrained isolated chimpanzee under entrained and free-running conditions. Aerospace Med 43:155-161. 
Meijer JH, Rietveld WJ (1989) Neurophysiology of the suprachiasmatic circadian pacemaker in rodents. Physiol Rev 69:671-707.

Mistlberger RE, Rusak B (1989) Mechanisms and models of the mammalian circadian system. In: Principles and practice of sleep medicine (Kryger MH, Roth T, Dement WC, eds), pp 141-152. Philadelphia: Saunders.

Mistlberger RE, Bergmann BM, Waldenar W, Kechtschaffen A (1983) Recovery sleep following sleep deprivation in intact and suprachiasmatic nuclei-lesioned rats. Sleep 6:217-233.

Moore RY (1983) Organization and function of a central nervous system oscillator: the suprachiasmatic hypothalamic nucleus. Fed Proc 42:2783-2789.

Moore-Ede MC, Sulzman FM, Fuller CA (1982) The clocks that time us. Physiology of the circadian timing system. Cambridge, MA: Harvard UP.

Nauta WJH (1946) Hypothalamic regulation of sleep in rats. An experimental study. J Neurophysiol 9:285-315.

Newman GC, Hospod FE (1986) Rhythm of suprachiasmatic nucleus 2-deoxyglucose uptake in vitro. Brain Res 381:345-350.

Obal F, Benedek G, Obal F, Dibo G, Jancso-Gabor A (1982) Basal forebrain sleep mechanisms activated by electrical and thermal stimulations. In: Motivation and the neural and neurohumoral factors in regulation and behaviour, Vol 10, Recent developments of neurobiology in Hungary (Lissak K, Molnar P, eds), pp 159-175. Budapest: Akademiai Kiado.

Ralph MR, Lehman MN (1991) Transplantation in the analysis of the mammalian hypothalamic circadian pacemaker. Trends Neurosci 14:362-366.

Ralph MM, Foster RG, Davis FC, Menaker M (1990) Transplanted suprachiasmatic nucleus determines circadian period. Science 247 975-978.

Reppert SM, Perlow MJ, Underleider LG, Mishkin M, Tamarkin L, Orloff DG, Hoffman HJ, Klein DC (1981) Effects of damage to the suprachiasmatic area of the anterior hypothalamus on the daily melatonin and cortisol rhythms in the rhesus monkey. J Neurosci 1:1414 1425.

Richardson GS, Carskadon MA, Flagg W, Van den Hoed J, Dement WC, Mitler MM (1978) Excessive daytime sleepiness in man: multiple sleep latency measurement in narcoleptic and control subjects. Electroencephalogr Clin Neurophysiol 45:621-627.

Richardson GS, Carskadon MA, Orav EJ, Dement WC (1982) Circadian variation of sleep tendency in elderly and young subjects. Sleep 5.S82-S94.

Richardson GS, Moore-Ede MC, Czeisler CA, Dement WC (1985) Circadian rhythms of sleep-wakefulness in mice: analysis using longterm automated recordings of sleep. Am J Physiol 248:R320-R330.

Richter CP (1968) Inherent twenty-four hour and lunar clocks of a primate - the squirrel monkey. Commun Behav Biol 1A:305-332.

Richter CP (1971) Inborn nature of the rat's 24-hour clock. J Comp Physiol Psychol 75:1-4.

Roehrs T, 7wyghuizen-Doorenhos A, Timms V, Zorick F, Roth T (1989) Sleep extension, enhanced alertness and the sedating effects of ethanol. Pharmacol Biochem Behav 34:321-324.

Rummel M, Lee JK, Halberg F (1974) Combined linear-nonlinear chronobiologic windows by least squares resolve neighbouring components in a physiologic rhythm spectrum. In: Biorhythms and human reproduction (Ferin M, Halberg F, Richart RM, Vande Wiele RL, eds), pp 53-82. New York: Wiley.

Rusak B (1977) The role of the suprachiasmatic nuclei in the generation of circadian rhythms in the golden hamster. J Comp Physio [A] 118:145-165

Rusak B, Zucker I (1979) Neural regulation of circadian rhythms. Physiol Rev 59:449-526.

Satinoff E, Prosser R (1988) Suprachiasmatic nuclear lesions eliminate circadian rhythms of drinking and activity, but not of body temperature, in male rats. $J$ Biol Rhythms 3:1-22.

Sato $T$, Kawamura H (1984a) Circadian rhythms in multiple unit activity inside and outside the suprachiasmatic nucleus in the diurnal chipmunk (Eutamias sibiricus). Neurosci Res 1:45-52.

Sato T, Kawamura H (1984b) Effects of bilateral suprachiasmatic nucleus lesions on the circadian rhythms in a diurnal rodent, the Siberian chipmunk (Eutamias sibiricus). J Comp Physiol [A] 155 745-752.

Schwartz WJ, Gainer, H (1977) Suprachiasmatic nucleus: use of ${ }^{14} \mathrm{C}$ - labeled deoxyglucose uptake as a functional marker. Science 197: 1089-1091.

Schwartz WJ, Reppert SM, Eagan SM, Moore-Ede MC (1983) In vivo metabolic activity of the suprachiasmatic nuclei: a comparative study. Brain Res 274:184-187.

Seidel WF, Cohen SA, Bliwise NG, Roth T, Dement WC (1986) Doserelated effects of triazolam and flurazepam on a circadian rhythm insomnia. Clin Pharmacol Ther 40:314-320.

Sheatz GC (1961) Electrode holders in chronic preparation. In: Electrical stimulation of the brain (Sheer DE, ed), pp 45-50. Austin, TX: University of Texas.

Sofroniew MV, Weindl A (1982) Neuroanatomical organization and connections of the suprachiasmatic nucleus. In: Vertebrate circadian systems: structure and physiology (Aschoff J, Daan S, Groos G, eds), pp 75-86. Berlin: Springer.

Sokolove PG, Bushell WN (1978) The chi-square periodogram: its utility for analysis of circadian rhythms. J Theor Biol 72:131-160.

Stephan FK, Swann JM, Sisk CL (1979) Anticipation of 24-hr feeding schedules in rats with lesions of the suprachiasmatic nucleus. Behav Neural Biol 25:346-363.

Stephan FK, Berkley KJ, Moss RL (1981) Efferent connections of the rat suprachiasmatic nucleus. Neuroscience 6:2625-2641.

Steriade M (1989) Brain electrical activity and sensory processing during waking and sleep states. In: Principles and practice of sleep medicine (Kryger MH, Roth T, Dement WC, eds), pp 86-103. Philadelphia: Saunders.

Steriade M, Llinas RR (1988) The functional states of the thalamus and associated neuronal interplay. Physiol Rev 68:649-742.

Steriade M, McCarley RW (1990) Brainstem control of wakefulncss and sleep. New York: Plenum.

Sterman MB, Clement CD (1974) Forebrain mechanisms for the onset of sleep. In: Basic sleep mechanisms (Petre-Quadens O, Schlag J, eds), pp 83-97. New York: Academic.

Sterman MB, Shouse MN (1985) Sleep "centers" in the brain: the preoptic basal forebrain area revisited. In: Brain mechanisms of sleep (McGinty DJ, Drucker-Colin R, Morrison A, Parmeggiani PL, eds), pp 277-299. New York: Raven.

Strogatz SH (1986) Lecture notes in biomathematics. The mathematical structure of the human sleep-wake cycle, Vol 69 (Levin S, ed), pp 1-239. New York: Springer.

Strogatz SH, Kronauer RE, Czeisler CA (1986) Circadian regulation dominates homeostatic control of sleep length and prior wake length in humans. Sleep 9:353-364.

Strogatz SH, Kronauer RE, Czeisler CA (1987) Circadian pacemaker interferes with sleep onset at specific times each day: role in insomnia. Am J Physiol 253:R172-R178.

Szymusiak R, Satinoff E (1984) Ambient temperature dependence of sleep disturbances produced by basal forebrain damage in rats. Brain Res Bull 12:295-305.

Tobler I, Borbely AA (1986) Sleep EEG in the rat as a function of prior waking. Electroencephalogr Clin Neurophysiol 64:74-76.

Tobler I, Borbely AA, Groos G (1983) The effect of sleep deprivation on sleep in rats with suprachiasmatic lesions. Neurosci Lett 42:4954.

Tokura H, Aschoff J (1978) Circadian activity rhythm of the pigtailed macaque (Macaca nemestrina) under constant illumination. Pfluegers Arch 376:231-243.

Trachsel L, Edgar DM, Seidel WF, Heller WC, Dement WC (1992) Sleep homeostasis in suprachiasmatic nuclei-lesioned rats: effects of sleep deprivation and triazolam administration. Brain Res 589:253261.

Van den Pol AN (1991) The suprachiasmatic nucleus: morphological and cytochemical substrates for cellular interaction. In: Suprachiasmatic nucleus: the mind's clock (Klein DC, Moore RY, Reppert SM, eds), pp 17-50. New York: Oxford UP.

Von Economo C (1931) Encephalitis lethargica: its sequelae and treatment. London: Oxford UP.

Watts AG (1991) The efferent projections of the suprachiasmatic nucleus: anatomical insights into the control of circadian rhythms. In: Suprachiasmatic nucleus: the mind's clock (Klein DC, Moore RY, Reppert SM, eds), pp 77-106. New York: Oxford UP.

Watts AG, Swanson LW, Sanchez-Watts G (1987) Efferent projections of the suprachiasmatic nucleus. I. Studies using anterograde transport of Phaseolus vulgaris leucoagglutinin in the rat. J Comp Neurol 258: 204-229. 
Welsh DK, Richardson GS, Dement WC (1988) Effect of running wheel availability on circadian patterns of sleep and wakefulness in mice. Physiol Behav 43:771-777.

Wexler DB, Moore-Ede MC (1985) Circadian sleep-wake cycle organization in squirrel monkeys. Am J Physiol 248:R353-R362.
Wong-Riley M (1979) Changes in the visual system of monocularity sutured or enucleated cats demonstrable with cytochrome oxidase histochemistry. Brain Res 171:11-28. 